\title{
34 System-on-Chip RF Sensors for Life and Geo Sciences
}

\author{
D. Zito, F. Alimenti, D. Pepe, A. Fonte, F. Zito, A. Lanatà, and D. De Rossi
}

\section{Introduction}

In the framework of ongoing technology trends, well-known systems traditionally implemented using hybrid technologies (that are bulky and expensive) have interesting new possibilities. Novel miniaturized sensors are expected to lie at the crossroads between information communication technology and the life and geo-sciences. Interaction between human beings and the physical environment and/or other entities will be possible through the availability of sophisticated micro/nano-systems.

Today, microelectronic systems in standard silicon technologies are capable of operation with radio-frequency $(\mathrm{RF})$ signals up to a few hundred $\mathrm{GHz}$. In addition to traditional data communication (e.g. mobile phones, wireless local area networks, etc), RF signals could be exploited also for contactless sensing and detection of information from the environment including the vital signs of human beings. Despite the differences among the possible applications, they can all be grouped under the same discipline of advanced radio-frequency engineering.

In this chapter we present two innovative contributions derived by the System-on-Chip (SoC) approach combining standard microelectronic technologies with the life and geo-sciences. The former regards a $\mathrm{SoC}$ radar sensor for contactless cardiopulmonary monitoring. The latter focuses on a SoC radiometer for temperature remote sensing. For both micro-sensors we report the framework and motivation, theoretical concepts, current results, and the future direction of ongoing research by the authors.

\section{SoC Radar Sensor for Cardio-Pulmonary Monitoring}

The evolution of the wireless technology is supported by communication standard innovations. In particular, it is worth mentioning that in February 2002 the Federal Communications Commission (FCC) gave permission for the marketing and operation of a new class of products incorporating ultra-wideband (UWB) technology [1]. The FCC, through a modification of the 47 CRF Part 15 regulations [2], decided to allocate, for the first time and in a non-exclusive way, an unlicensed $7.5 \mathrm{GHz}$ band in the frequency range 3.1-10.6 GHz. The FCC imposed a very low maximum power spectral density (PSD) of $-41.3 \mathrm{dBm} / \mathrm{MHz}$ over the entire band since such UWB systems operate in regions of spectrum in which other services are already operating. Asia and Europe have recently adopted similar regulations for the UWB systems in the same frequency band $[3,4]$.

Traditional RF wireless systems transmit information by means of one or more continuous wave modulated carriers. The basic concept of UWB systems is to transmit and receive extremely short RF pulses with a time duration in the range from tens of picoseconds to tens of nanoseconds. For this reason, such signals will result in very wide frequency content and an extremely low power spectral density.

This new technology is devoted to the realization of very low power and high data rate wireless communication systems. However, one of the most promising applications of UWB technology is low-cost medical imaging. In fact, a UWB radar sensor can be employed to monitor vital parameters 
non-invasively and without any contact, as well as the heart-wall and thorax movements, by detecting in real-time the heart and breath rates, respectively.

In particular, the first part of this chapter (sections 3-8) is focused on this opportunity to realize a novel wearable wireless sensor interface for the monitoring of the heart beat and breath rates. Most of the contents highlighted hereinafter are derived from research related to the ProeTEX project (FP6-2004-IST-4-026987), a European Integrated Project aimed at developing a new generation of equipment for emergency personnel such as firefighters and civil protection rescuer.

By considering the latest advances in modern silicon technology such as 90-nm CMOS consisting of devices with cut-off frequencies higher than $150 \mathrm{GHz}$, we target the realization of such a lowcost, miniaturized, very low-power wireless UWB sensor. This innovative system-on-chip device could extend the capability of the Wireless Body Area Network (WBAN) from wireless connectivity around the body to a new class of applications based on personal RF sensing.

In particular, we focus on the design of an innovative fully integrated 3.1-10.6 GHz UWB radar for cardiopulmonary monitoring. In section 3 the use of radar systems for medical imaging is introduced. In section 4 the system overview and its operating principle are discussed. In section 5 a theoretical study on the propagation channel model of UWB pulses into the human body is presented. In sections 6 and 7 the theoretical and CAD system analyses are reported, respectively.

\subsection{Radar in Medicine}

Microwave Doppler radar has been used to detect the respiration rate since 1975 [5]. These first devices were bulky and expensive, but recently fully integrated CMOS versions of a radar for noncontact cardiopulmonary monitoring have been presented [6]. Doppler radars transmit a continuous wave $(\mathrm{CW})$ signal and receive the echo reflected from the target. Such a radar system realizes the beating between the received wave and the replica of the transmitted one. The resulting signal will have a non-zero frequency if the target, which generates the echo, has a non-zero relative velocity with respect to the radar.

Another class of radar employed in the monitoring of vital parameters is pulse radar. Pulse radars operate by sending short electromagnetic pulses and by receiving the echoes reflected by the target. The time delay between the transmitted pulse and the received pulse is proportional to the distance from the target to the radar. Examples of pulse radars for the detection of vital parameters are reported in literature [7, 8]. The principle of operation consists of sending extremely short electromagnetic pulses towards the heart and receiving the echo reflected from the separation between the heart muscle and the blood that flows inside it, since the characteristic impedances of these two media are different.

There are great advantages to employing radar systems for the monitoring of vital parameters with respect to traditional methods. Radar systems allow for monitoring of heart activity non-invasively and without contact [9] unlike traditional techniques such as the electrocardiograph, the echocardiograph, and the pulsed oximetry. Indeed, the electrocardiograph requires the application of electrodes on the thorax. The echocardiograph requires the use of a gel between skin and probe as well as an expert operator. Pulsed oximetry requires the use of sensors applied on a finger or earlobe. Moreover, a radar system can directly monitor the heart wall movement instead of just its electrical activity (such as the electrocardiograph), allowing for the detection of hearth diseases that do not manifest as anomalies in electrical activity (i.e. arrhythmias).

UWB transceivers present a lower complexity with respect to traditional RF systems from a circuit implementation point of view thus leading to low power consumption and a longer life battery. In fact, with respect to the electrocardiograph, UWB systems do not require a stable frequency reference, which typically requires a large area on silicon die and is power hungry. Last but not least, the extremely low level of transmitted power density (lower than $-41.3 \mathrm{dBm} / \mathrm{MHz}$ ) of the UWB radar should reduce the risk of molecular ionization [10-15]. 


\subsection{UWB Radar for Heart Wall Monitoring: System Overview and Operating Principle}

The system concept is shown in Figure 1. It consists of a fully integrated UWB radar sensor and lowpower radio interface. The radar sensor and the low data-rate wireless transceiver will be implemented in a standard 90-nm CMOS technology by ST Microelectronics. Each antenna is realized on a microstrip substrate. However, in a most advanced realization they can be integrated directly into the clothes by means of conductive tissues [16]. These interfaces will be inserted into an inner garment worn by emergency operators [17]. The data acquired by the UWB radar sensor will be transferred to a personal or remote data acquisition and signal processing unit by means of the low-power radio data link realized by a wireless transceiver based on the IEEE 802.15.4 (ZigBee) standard. This can even be sent directly to medical staff which can monitor in real time the person under observation (see Figure 2). By means of the low-power transceiver the UWB radar sensor can be remotely programmed, thus increasing the system flexibility.

Figure 3 depicts the architecture for the radar system. A correlator-based receiver has been adopted for implementation in modern silicon technology since the cross-correlator has a frequency response equal to that of a matched filter. In particular, it can be demonstrated that the matched filter allows us to obtain the best signal-to-noise ratio at the output [18]. Preliminary simulations have shown that this topology allows us to achieve the best performance in terms of output signal-to-noise ratio (SNR) and sensitivity to small variations of the position of the heart wall with respect to other topologies, like that in which the receiver is simply turned on by the command given by the delayed replica of the transmitted pulse $[7,8]$.

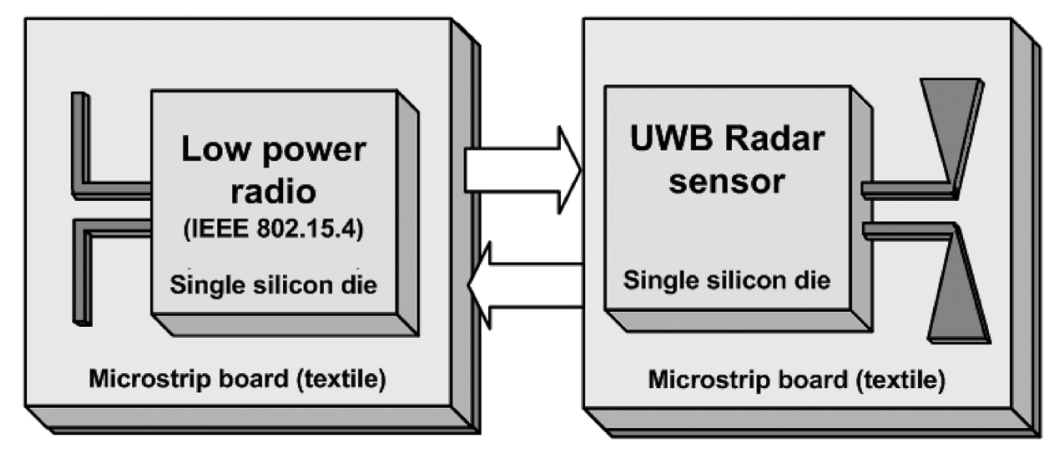

(a)

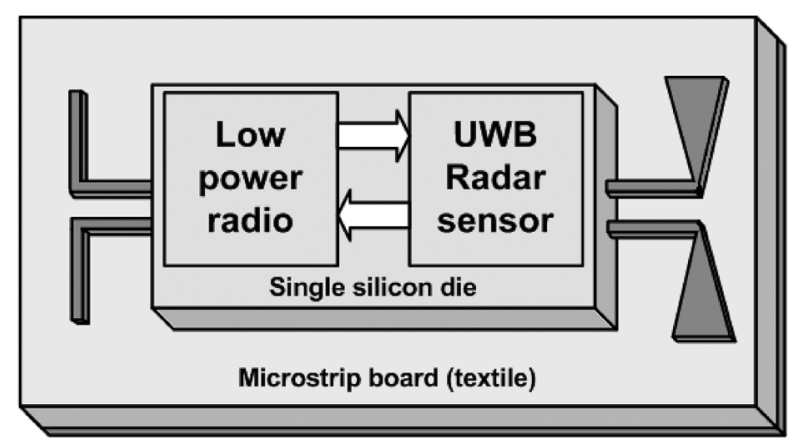

(b)

FIGURE 1 Wearable wireless radio interface for monitoring of the cardiopulmonary activity: (a) system concept; (b) future perspective: integration on a single silicon chip of both the low power ZigBee transceiver and the UWB Radar. 


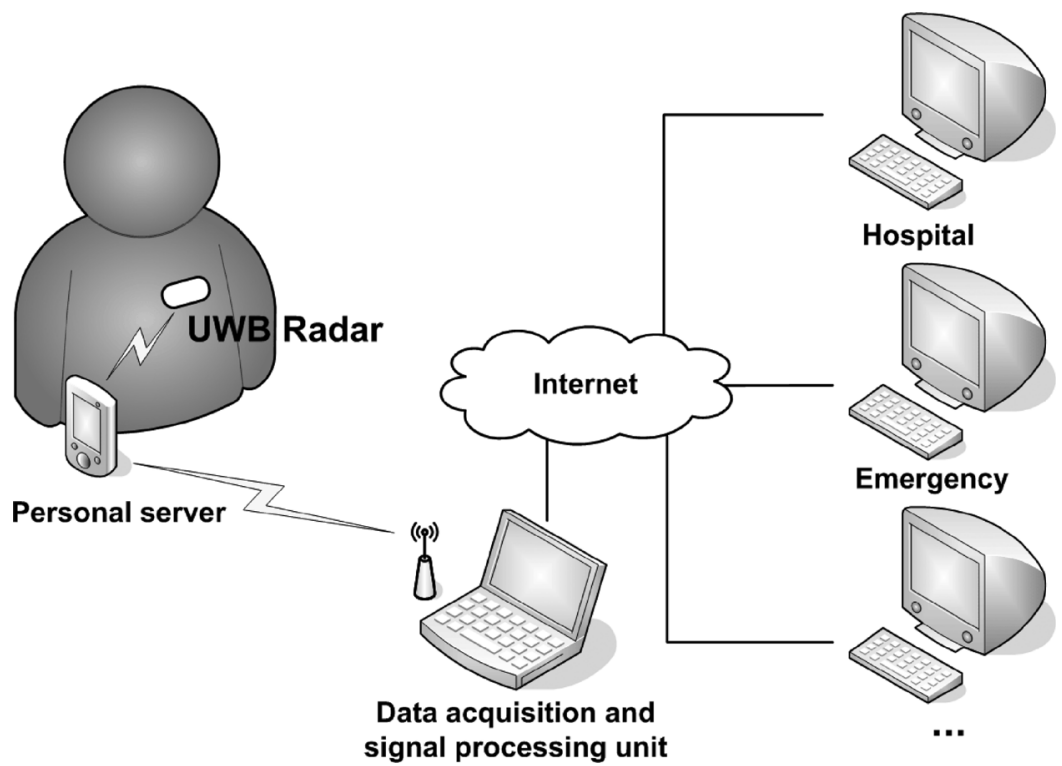

FIGURE 2 Networking scenario for the UWB radar sensor.

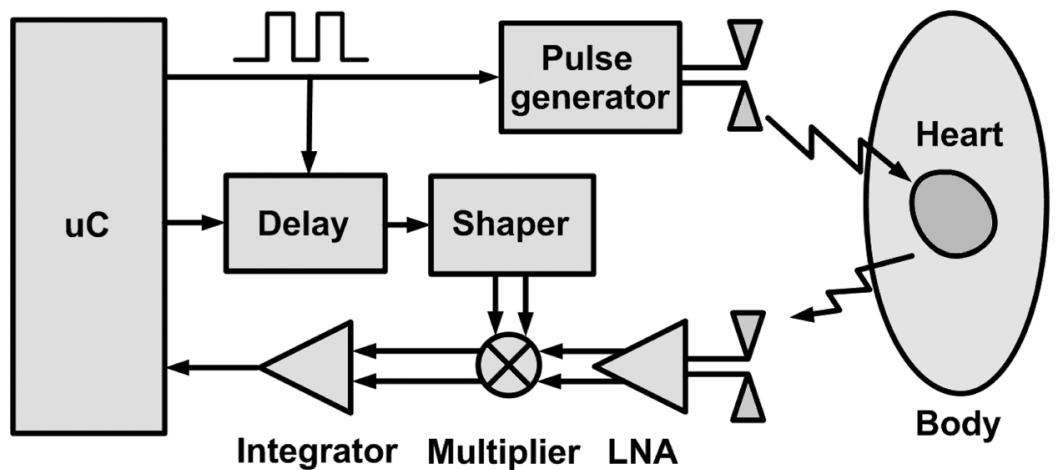

FIGURE 3 Block diagram of the fully integrated UWB radar sensor.

The operating principle of the cross-correlator radar is as follows. The pulse generator transmits a train of extremely short (the time duration should be around 200-400 ps) electromagnetic pulses toward the target (the heart). Since the heart muscle and the blood that flows inside have different characteristic impedance, part of the energy of the transmitted pulses is reflected at the interface of these two different media [7]. A pulse repetition frequency (PRF) greater than $1 \mathrm{MHz}$ allows us to consider the heart almost "motionless" between two consecutive pulses. After a time delay approximately equal to the flight time of the pulse from the transmitter to the receiver (about two nanoseconds), the echoes received from the target are multiplied with a delayed local replica of the transmitted pulses generated by the Shaper block (see Figure 3). If the time delay is fixed, a fixed range gate is monitored by the radar, and the amplitude of the signal at the output of the multiplier is related to the position of the heart. The output signal will reach its maximum in the case of perfect time alignment between the two signals at the input of the multiplier itself. The output signal of the multiplier is then integrated. The integrator averages a large number of pulses order to increase the signal-to-noise ratio and to have an output signal that contains the frequencies of the vital parameters under observation. Since the vital parameters vary with frequencies on the order of one Hertz, an 
integrator bandwidth of $100 \mathrm{~Hz}$ will be large enough to properly detect them. The output of the integrator will contain both the heart beat and the breath tones, since the distance from the radar to the heart wall varies both with the heart contraction-expansion and with inhalation-exhalation of breath. Measurements with a discrete pulse radar for the heart monitoring reported in literature [10] show this result.

One of the most relevant tasks at this stage of the study consists of deriving a descriptive model of the human chest, which could allow us to carry out a complete system analysis and its verification by means of system simulation. In fact, the pulses transmitted will be strongly attenuated in the human chest and their shape will be modified since the spectral components of the signal will be attenuated differently.

\subsection{Channel Propagation Model}

A proper channel model has to be developed in order to carry out system analyses and system simulations. The transmitted pulses will be strongly attenuated in the human chest. Moreover, the shape of the pulse will be modified since the spectral components of the signal will be attenuated differently. In order to perform system simulations, it is also important that the channel model takes into account the variation of the flight time of the pulses from the transmitter to the receiver due to the periodic variation of the distance from the radar to the heart wall.

The first objective is to find the losses in the human chest in all the range of frequencies of operation. A frequency dependent channel loss model (in the band 1-12 GHz) has been developed. The overall loss $L(f)$ in $\mathrm{dB}$ has been calculated by taking into consideration the contributions of: (1) path loss $P L(f),(2)$ attenuation in the tissues $A t t(f)$, and (3) losses due to the reflections at the interface between different tissues $R f(f)$ as

$$
L(f)=P L(f)+\operatorname{Att}(f)+R f l(f) .
$$

The dielectric characteristic of human tissue has been extracted by the parametric model developed by C. Gabriel and colleagues at the Brooks Air Force Base (U.S.A.) $[19,20]$. As for the thickness of the tissue layers, the model proposed in [9], based on the Visible Human Project and Gabriel's data book, has been considered. In Table 1 the layers considered and their thickness are reported. Since we rely on applying the system-on-a-chip radar (and then its antenna) very close to the skin in proximity of the heart region (wearable system), near field equations $[21,22]$ have been used in order to evaluate the path and reflection losses.

As for path loss, the far-field power decreases as the inverse square of the distance $\left(1 / r^{2}\right)$. In the near-field range, the power falls down faster, typically as the inverse fourth $\left(1 / r^{4}\right)$ or higher of the

TABLE 1 Chest layer thickness considered for the system analysis.

\begin{tabular}{lc}
\hline Layers & Thickness [mm] \\
\hline air & 10 \\
fat & 9.6 \\
muscle & 13.5 \\
cartilage & 11.6 \\
lung & 5.78 \\
heart wall & 5 \\
\hline
\end{tabular}


distance. The path loss term has been estimated as follows

$$
\begin{aligned}
P_{R X} & =\frac{P_{T X} G_{T X} G_{R X}}{4}\left(\frac{1}{(2 k r)^{2}}-\frac{1}{(2 k r)^{4}}+\frac{1}{(2 k r)^{6}}\right) \\
& =\frac{P_{T X} G_{T X} G_{R X}}{4}\left(\left(\frac{\lambda_{m}(f)}{4 \pi r}\right)^{2}-\left(\frac{\lambda_{m}(f)}{4 \pi r}\right)^{4}+\left(\frac{\lambda_{m}(f)}{4 \pi r}\right)^{6}\right)
\end{aligned}
$$

where $G_{T X}$ is the transmitter antenna gain, $G_{R X}$ is the receiver antenna gain, $\lambda_{m}$ is the average wavelength of the electromagnetic radiation in the tissues of the human chest, $k$ is the wave number, $r$ is the distance from the radar to the heart, and $f$ is the frequency. The term $2 r$ takes into account the two-way transit. An antenna gain $\left(G_{T X}=G_{R X}\right)$ equal to $1.8(2.5 \mathrm{~dB})$ has been chosen to calculate the contribution of the path loss by referring to the UWB antenna measured and proposed in [23].

The attenuation in the tissues has been calculated as

$$
\operatorname{Att}(f)=\prod_{i=0}^{5} \exp \left(-\frac{2 r_{i}}{\delta_{i}(f)}\right)
$$

where $r_{i}$ and $\delta_{i}$ are the thickness and the penetration depth of the $i^{\text {th }}$ layer between the antenna and the heart muscle (index 0 refers to the air, index 1 to fat, index 2 to muscle, index 3 to cartilage, index 4 to lung, and index 5 to the heart muscle). As in equation (4), $2 r_{i}$ takes into account the two-way path loss.

The loss due to the reflections $R f(f)$ has been evaluated by taking into account the signal transmitted at each interface, both in the forward path and return path (equations (4) and (5), respectively), and the signal reflected at the heart/blood interface (6) in $\mathrm{dB}$

$$
\begin{aligned}
\tau_{1} & =\prod_{i=0}^{4} \frac{2 Z_{W, i+1}(f)}{Z_{W, i+1}(f)+Z_{W, i}(f)} \\
\tau_{2} & =\prod_{i=0}^{4} \frac{2 Z_{W, i}(f)}{Z_{W, i+1}(f)+Z_{W, i}(f)} \\
\Gamma_{5,6} & =\frac{Z_{W 6}(f)-Z_{W 5}(f)}{Z_{W 6}(f)+Z_{W 5}(f)} \\
R f l(f) & =\tau_{1}(f)+\Gamma_{5,6}(f)+\tau_{2}(f)
\end{aligned}
$$

where $Z_{W, i}$ is the wave impedance estimated for the $i^{\text {th }}$ layer between the antenna and the heart/blood interface (the index 6 refers to the blood), calculated for the near-field range [22] as

$$
Z_{W, i}=\frac{E_{\theta}}{H \phi}=\xi_{i} \frac{\frac{j}{k_{i} r_{i}}+\frac{j}{\left(k_{i} r_{i}\right)^{2}}-\frac{j}{\left(k_{i} r_{i}\right)^{3}}}{\frac{j}{k_{i} r_{i}}+\frac{j}{\left(k_{i} r_{i}\right)^{2}}}
$$

where $\xi_{i}$ is the characteristic impedance of the $i^{\text {th }}$ layer for the far-field range.

Due to the high conductivity of body tissue, attenuation loss is the most relevant contribution of those described above, especially at high frequency. The overall channel loss evaluated from 1 to $12 \mathrm{GHz}$ is shown in Figure 4. Simulation results show that the average power loss of the pulse in the 3.1-10.6 GHz band amounts to about $80 \mathrm{~dB}$.

Regarding the time-of-flight $\left(t_{o f}\right)$ of the electromagnetic pulse from the radar antenna to the heart and back, it has been estimated to be approximately 2 ns via

$$
t_{o f}=2 \prod_{i=0}^{5} \frac{r_{i}}{v_{i}} \approx 2 \mathrm{~ns}
$$




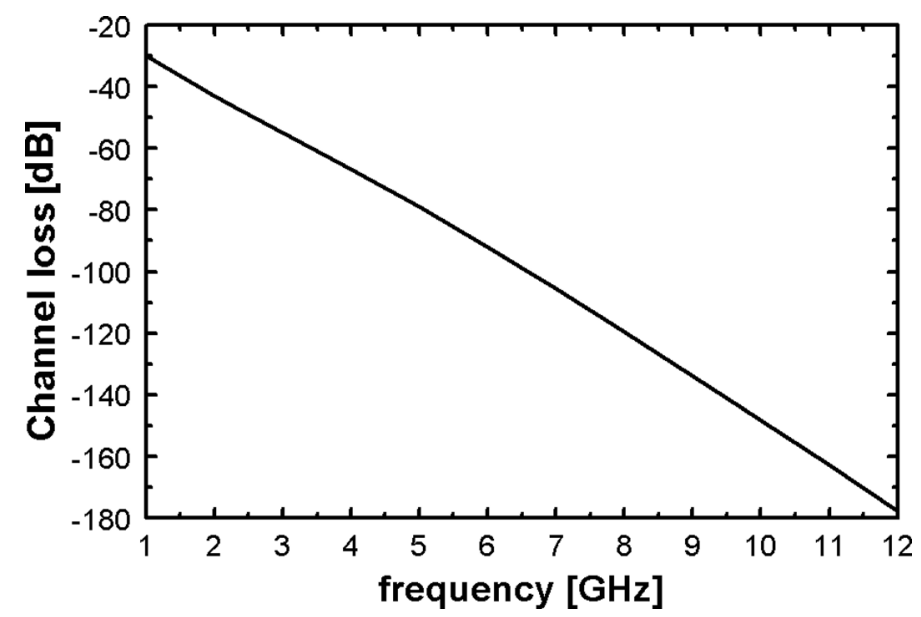

FIGURE 4 Channel loss vs. frequency of the model of intra-body channel.

where $r_{i}$ is the thickness of the $i^{\text {th }}$ layer (air, fat, muscle, cartilage, lung and heart) and $v_{i}$ is the speed of the electromagnetic radiation therein. This value is confirmed in the literature [7, 9].

With the hypothesis that the radar is motionless, the flight time of the electromagnetic pulse varies with the position of the heart wall. In [24] it is stated that the maximum displacement of the heart $(d)$ is $1.5 \mathrm{~cm}$. This implies that the maximum difference in the flight time $\left(\Delta T_{S M A X}\right)$ of the transmitted pulses amounts to

$$
\Delta T_{S M A X}=\frac{2 d}{\langle v\rangle} \approx 470 \mathrm{ps}
$$

where $\langle v\rangle$ is the mean speed of the radiation in the tissues.

\subsection{Theoretical System Analysis}

The transmitted electromagnetic pulses need to have very short time duration in order to achieve a range resolution on the order of a centimeter, since the maximum displacement of the heart wall is only a few centimeters. A pulse time duration $\tau$ of $\sim 200 \mathrm{ps}$ provides this degree of range resolution. By considering that the peak of the spectrum of a Gaussian monocycle pulse is at the frequency $1 / \tau$, the power spectral density for a 200 ps pulse train has a peak at $5 \mathrm{GHz}$ (i.e. within the $3.1-10.6 \mathrm{GHz}$ band).

The Gaussian monocycle is preferred to the Gaussian pulse because it does not have a DC component and its spectrum fits within the FCC emission mask. Furthermore, the Gaussian monocycle pulse can be implemented efficiently on silicon by means of a differential transmitter [25]. For system analysis the peak-to-peak voltage of the pulse has been chosen to be $1.2 \mathrm{~V}$ based on the radar being realized in a standard 90-nm CMOS technology by ST Microelectronics, which is characterized by a power supply of $1.2 \mathrm{~V}$ (this is not a critical value since the theoretical maximum peak-to-peak voltage of the pulse generated by the differential transmitter amounts to double the supply voltage without considering the voltage drop in the transistors).

As for the receiver, the minimum power $S_{\min }$ of a received signal, for a given signal-to-noise-ratio at the output of the receiver $S N R_{\text {out }}$, can be estimated by means of the following equation

$$
S_{\min }=k \cdot T \cdot B \cdot N F \cdot S N R_{\text {out }}
$$

where $k$ is the Boltzmann's constant $\left(1.38 \times 10^{-23}\right), T$ is the equivalent temperature of the antenna, $N F$ is the receiver noise factor, and $B$ is the bandwidth of the receiver. If we consider an average 
channel loss equal to $80 \mathrm{~dB}$ in the $3.1-10.6 \mathrm{GHz}$ band a noise figure lower than $-11.5 \mathrm{~dB}$ would be required in order to have an output signal-to-noise-ratio greater than $10 \mathrm{~dB}$ (for a single pulse).

This result is clearly not reachable in practice. The output SNR of the radar receiver can be increased by integrating several pulses [26]. The characteristic frequencies of the vital parameters under observation are on the order of a few Hertz, thus an integrator bandwidth of $100 \mathrm{~Hz}$ is wide enough to preserve the heart and breath rates. The case of an integrator with a bandwidth of $1 \mathrm{KHz}$ and a PRF in the range of $1-10 \mathrm{MHz}$ has been investigated. For a PRF of $10 \mathrm{MHz}, 10^{4}$ pulses are averaged. In particular, integration over the $10^{4}$ pulses provides a signal-to-noise-ratio improvement $\left(S N R_{\mathrm{imp}}\right)$ at the output of the receiver of about $40 \mathrm{~dB}$, as shown in the tables reported in [26]. This result is confirmed by the rough estimation given by

$$
S N R_{\text {imp }} \approx 10 \cdot \log \left(\frac{P R F}{B}\right)=10 \cdot \log 10000=40 \mathrm{~dB}
$$

where $B$ is the bandwidth of the integrator. The receiver front-end (LNA and multiplier) specifications can thus be relaxed by the integration of a large number of pulses. In these conditions the noise figure of the receiver front-end has to be lower than

$$
N F_{\text {max }}=-11.5+40=28.5 \mathrm{~dB} .
$$

The LNA is specified to have a power gain of $15 \mathrm{~dB}$ and a noise figure of $5 \mathrm{~dB}$, whereas for the multiplier has a voltage gain of $0 \mathrm{~dB}$ and a noise figure of $10 \mathrm{~dB}$. With these specifications, the noise figure of the overall receiver front-end is lower than the maximum NF allowed for a proper detection. It is worth mentioning that the aforementioned specifications can be obtained with standard $90-\mathrm{nm}$ CMOS technology.

In order to evaluate the amplitude of the output signal, the following hypotheses are employed. The first is that the input and output impedance match for the LNA. The second hypothesis is that the shape of the electromagnetic pulse does not vary in the path from the transmitter to the receiver (this hypothesis is hard to achieve but allows us to describe the radar system by means of simple mathematical equations).

Given the specifications for the LNA and multiplier above combined with an $80 \mathrm{~dB}$ channel loss, the pulse peak voltage at the output of the LNA is found to be $65 \mathrm{~dB}$ (i.e. $80-15)$ lower than the peak voltage of the transmitted pulse. Therefore, the peak voltage of the pulse at the output of the LNA is 1778 times (i.e. $65 \mathrm{~dB}$ ) lower than $0.6 \mathrm{~V}$ (i.e. about $337 \mu \mathrm{V}$ ).

The assumption of perfect time alignment between the pulse at the output of the LNA and the delayed local replica of the transmitted pulse allows us to calculate the maximum value of the signal expected at the output of the receiver. In particular, we suppose that the pulse at the input of the multiplier is multiplied with a replica of itself with $1-\mathrm{V}$ peak voltage. By considering the input pulse approximately equal to a one-period sinusoidal signal, then the output of the multiplier can be approximated with the square of an arch of sine with the same peak voltage of the input signal. The mean value of this pulse over a period is

$$
\bar{V}_{\text {out-mult }} \approx 337 \cdot\left(\frac{2}{\pi}\right)^{2} \mu \mathrm{V}=136 \mu \mathrm{V} .
$$

The output voltage of the multiplier at very low frequency (these components will be amplified by the integrator) can be approximated as

$$
V_{D C-\text { mult }} \approx 136.8 \cdot 10^{-6} \cdot \tau \cdot P R F
$$

which amounts to $274 \mathrm{nV}$ when the PRF is $10 \mathrm{MHz}$ (pulse duration $\tau=200 \mathrm{ps}$ ). With such a value, a gain of $120 \mathrm{~dB}$ is required for the integrator in order to have an amplitude voltage of a few hundred milli-Volts at the output. 


\subsection{CAD System Analysis}

The first-order theoretical analysis represents the first step but this does not take into consideration secondary, but non-negligible, aspects related to the technology issues. For this reason, the overall radar system has been implemented and simulated by means of the Ptolemy simulator within the CAD tool ADS2005A ${ }^{\mathrm{TM}}$ by Agilent Technologies. By means of the Ptolemy simulator, the operating principle of the UWB radar can be verified properly in the time domain. Each block of the overall radar system has been implemented in the simulator by functional blocks which take into account their bandwidth limitations and noise contributions. The Ptolemy simulator also allows the co-simulation of different blocks (schematics, S-parameter files, MATLAB files, et al.), thus when the building blocks are realized their measured performance can be inserted in the worksheet in order to make a more realistic system simulation.

The channel model (of the human chest) has been implemented by a frequency dependent loss block, and an additional block realizing a variable (periodic) flight-time to emulate the movement of the heart wall (see Figure 5). In particular, the first block consists of a set of S-parameters which provide the behavior in terms of frequency response equivalent to the theoretical channel model derived in the Section 4. The second block emulates a two-position heart wall by providing a difference of 470 picoseconds in the arrival time of the signal received for the two positions.

For the sake of clarity, the wall moving period has been set as 20 milliseconds (note that this is faster than the period of the heart beat; the time has been compressed with respect to real heart movement in order to reduce the simulation time and alleviate the computational hardware resources). Moreover, an additional noise source (white thermal noise) has been included in the channel to take into account the antenna noise at the input of the receiver. Both the antennas of the transmitter and receiver have been implemented in the simulator by means of a $7.5 \mathrm{GHz}$ band filter (centered at $6.85 \mathrm{GHz}$, with a slope of $-20 \mathrm{~dB} /$ decade). The LNA block has a band of 3.1-10.6 GHz and an out-of-band frequency response slope of $-20 \mathrm{~dB} /$ decade.

A two-step simulation has been carried out because of the extremely different time constants of the RF and baseband parts of the radar. In fact, the RF part has to be simulated with a time-step of about one tenth of the pulse width (i.e. the duration time), whereas the baseband part requires a simulation time of at least several tens of milliseconds. A time step of $10 \mathrm{ps}$ has been adopted for the $\mathrm{RF}$ section. The integrator (with $120 \mathrm{~dB}$ gain and $1 \mathrm{KHz}$ bandwidth) has been split into three low-pass filters with gain equal to $40 \mathrm{~dB}$ and bandwidth equal to $10 \mathrm{MHz}, 100 \mathrm{KHz}$ and $1 \mathrm{KHz}$, respectively. Simulation results have shown a good agreement with the results obtained by the theoretical system analysis. In particular, the simulations show that the SNR increase from the output of the multiplier to the output of the integrator is about $40 \mathrm{~dB}$, as predicted by the theoretical system analysis. The power spectral density (PSD) of a pulse train with $\tau=200 \mathrm{ps}, 1.2$ Volt peak-to-peak amplitude, and PRF of $1 \mathrm{MHz}$, at the input and output of an antenna filter, are shown in Figure 6, along with the

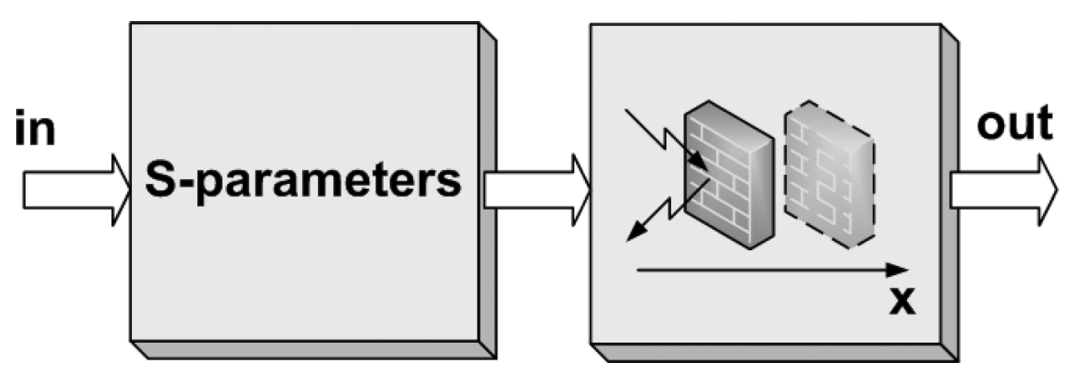

FIGURE 5 Simplified block diagram of the channel model implemented in ADS2005A. It consists of a S-parameter file (1-12 GHz) and a variable delay block that simulate two different positions of the heart-wall with respect to the radar. 


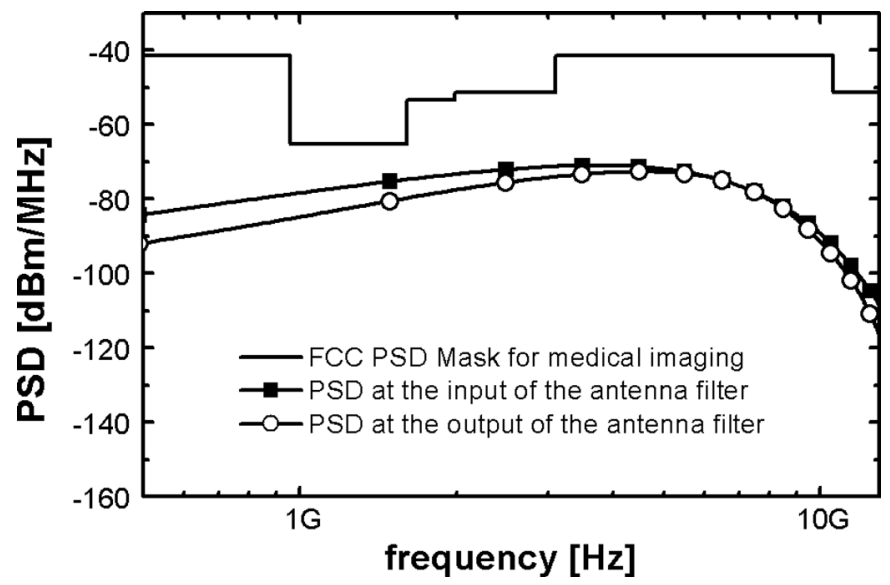

FIGURE 6 Power Spectral Densitiy (PSD) of a train of gaussian monocycle pulses of 200 ps and 1.2 Volts peakto-peak, with a PRF of $1 \mathrm{MHz}$, both at the input and output of the transmitter antenna filter.

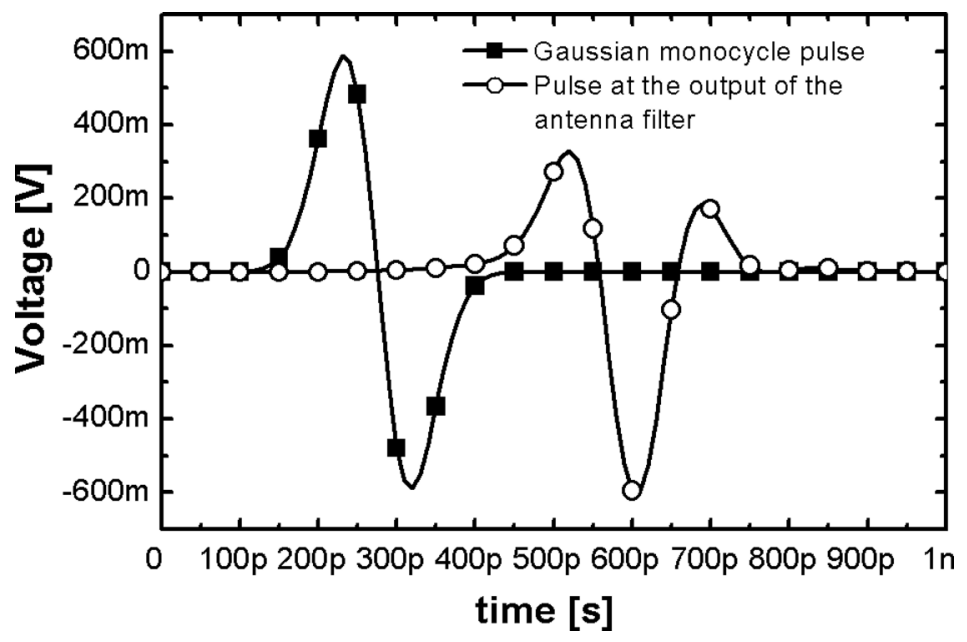

FIGURE 7 A 1.2-V peak-to-peak gaussian monocycle pulse at the input of the transmitter antenna filter, and the pulse at the output of the filter.

FCC mask for the UWB medical imaging applications. The 1.2-V peak-to-peak Gaussian monocycle pulse at the input of the transmitter antenna filter and the pulse obtained at the output of the antenna filter are shown in Figure 7. The pulse at the input of the receiver (at the output of the antenna filter of the receiver), in the presence of noise, is shown in Figure 8.

Note that the received pulse is distorted strongly by the human chest (i.e. the S-parameter block). In particular, the pulse is stretched out because the higher frequencies are attenuated the most. The output voltage of the integrator is shown in Figure 9. Note that the output signal reaches two different voltages for the two different positions of the heart. Finally, the CAD analysis has confirmed the feasibility derived by the theoretical study and enabled the next important step: the experimental verification of the channel model and the system-on-chip realization of the UWB radar sensor which is summarized in the following section. 


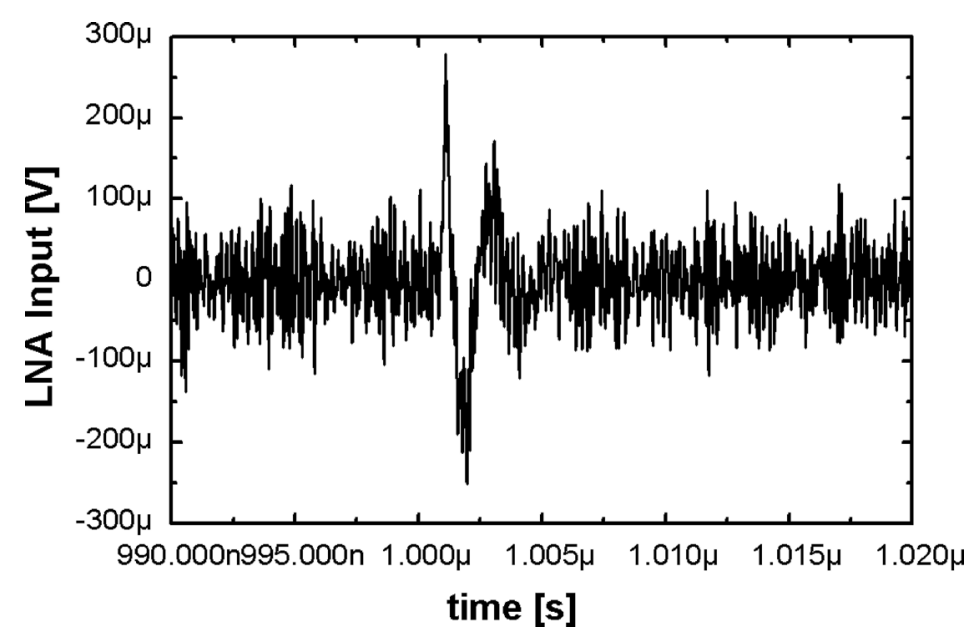

FIGURE 8 The input signal of the LNA (in presence of noise). Note that the pulse shape has been distorted strongly after the human-chest crossing.

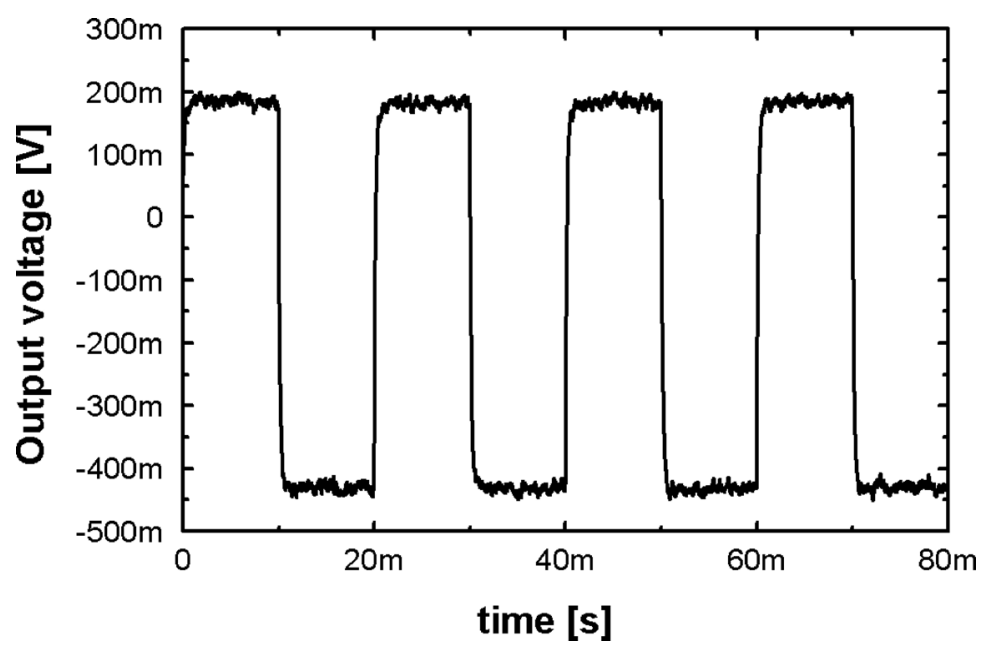

FIGURE 9 Output voltage of the integrator filter of the radar sensor. The output signal has the same frequency of the movement imposed for the heart wall. A time-varying surface with a period of $20 \mathrm{~ms}$ has been considered for the simulation.

\subsection{Present Status and Future Perspectives for the SoC UWB Radar}

The implementation of the UWB radar sensor for cardio-pulmonary monitoring has been scheduled into the two typical steps: (1) the design and the integration of the building blocks, and (2) the overall system-on-chip integration. Such an approach offers the designer an additional debugging phase consisting of the experimental verification of the circuit performance obtained at the end of the post-layout design phase. This margin could eventually be exploited to re-design each block to be compliant with specifications derived at the system-level design. When all the building blocks exhibit performance within the specifications they can be then integrated in a complete system-on-chip.

As of Q3/2008, the channel model has been verified experimentally and each building block of the overall radiometer has been designed and realized in a 90-nm CMOS standard process by ST-Microelectronics. The details regarding the successful channel model verification and the circuit 


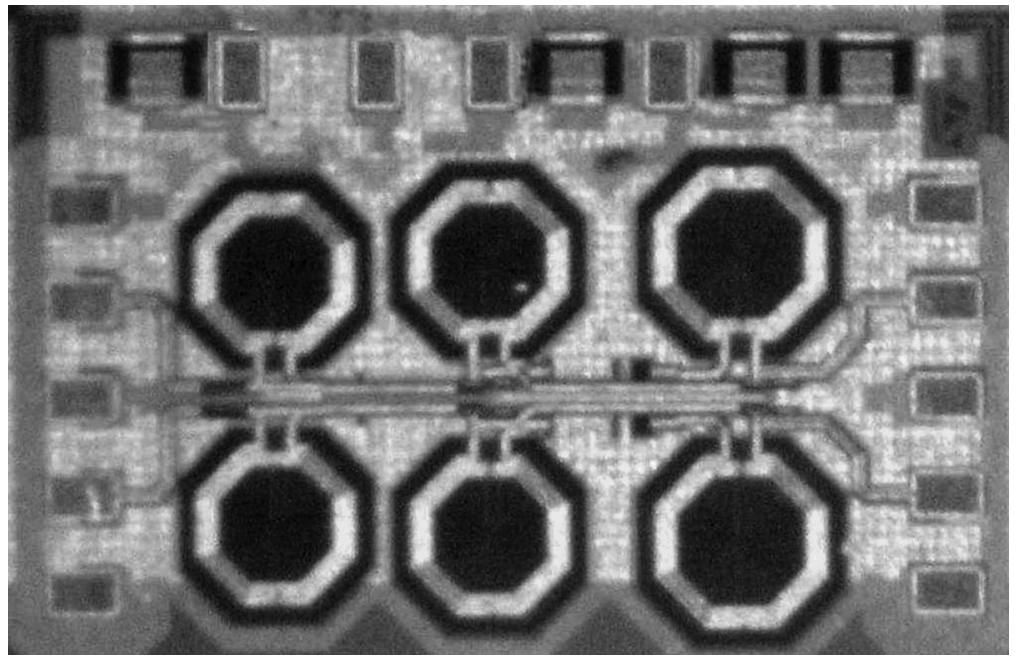

FIGURE 10 LNA testchip. The area size on die amounts to $0.68 \mathrm{~mm}^{2}$ (pads inclusive).

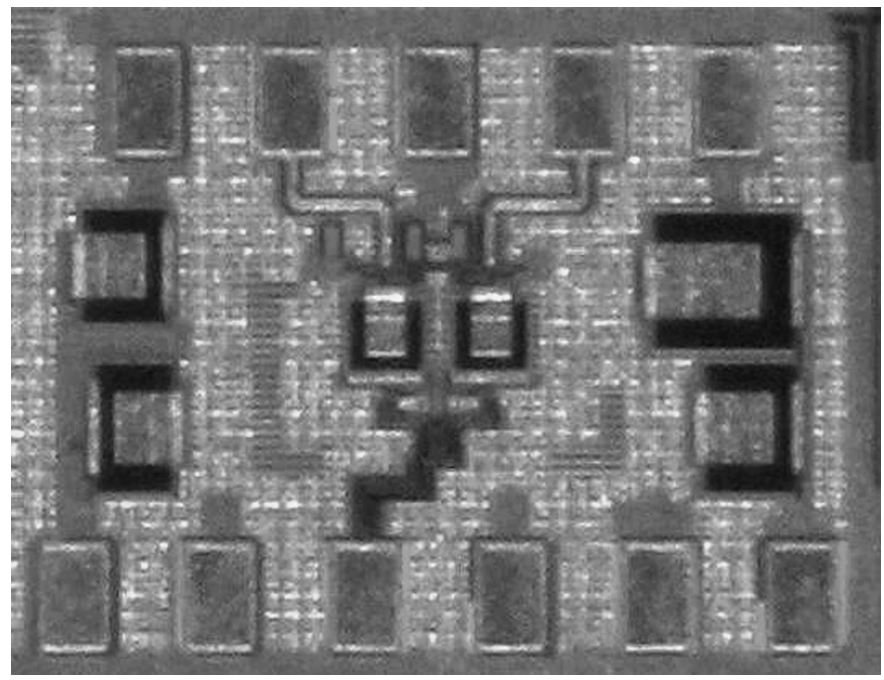

FIGURE 11 Pulse Generator testchip. The area size on die amounts to $0.27 \mathrm{~mm}^{2}$ (pads inclusive).

implementation can be found in [27]. The most critical building blocks (Low Noise Amplifier, Pulse Generator) have been tested successfully. As for the Multiplier, the Integrator, and the Delay Generator, the experimental characterization is in progress and the overall system-on-chip integration is scheduled for the Q1/2009. Below are the micrographs of the LNA and Pulse Generator test-chips (Figs. 10 and 11, respectively), and their performance (see Figs. 12 and 13). It is worth mentioning that the measurements have shown an almost perfect agreement with post-layout simulations.

Future work will address the integration of the UWB radar transceiver in a single silicon die, experimental characterization of the test-chip, its co-integration into a pc-based acquisition interface, and the overall sensor interface validation in a clinical environment. If the results of such medical tests are positive, the innovative system-on-chip UWB radar discussed herein will represent a new and decisive step forward to the realization of next-generation wireless sensors for human health-care portable systems, particularly for telemedicine applications. 


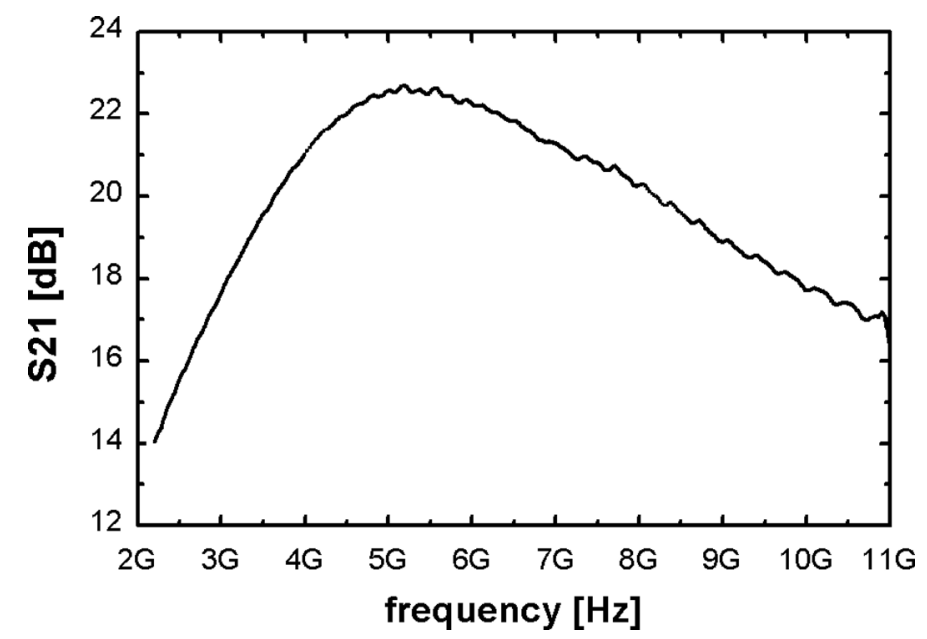

FIGURE 12 LNA power gain vs. frequency (measured).

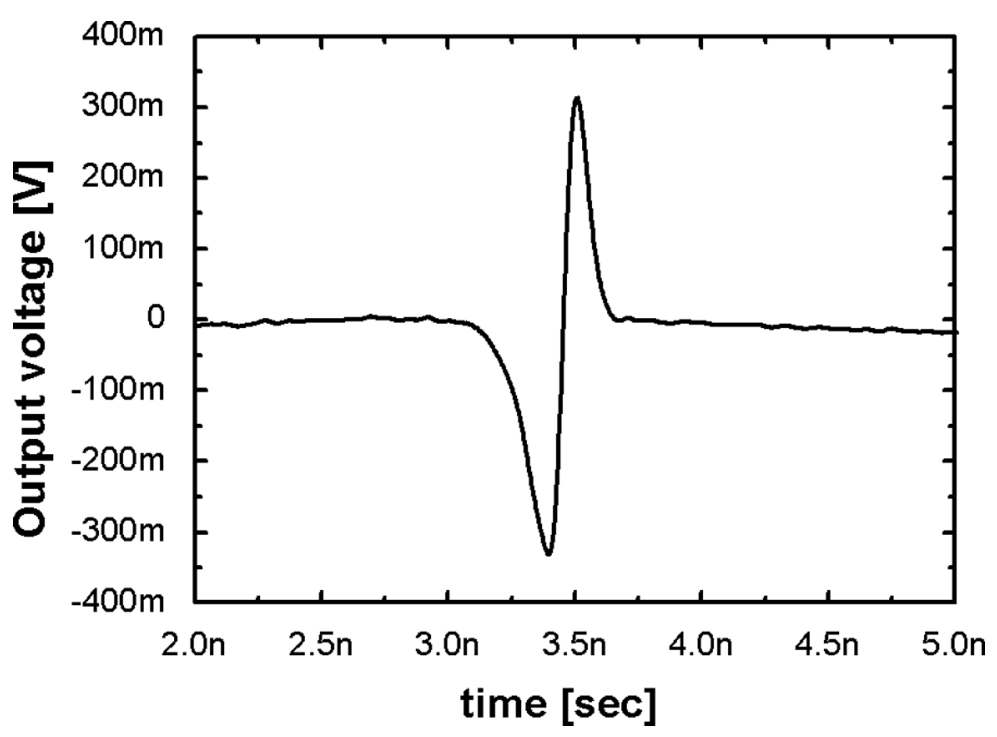

FIGURE 13 Output voltage pulse (measured).

\section{SoC Radiometer for Temperature Remote Sensing}

We now turn out attention to the application of system-on-chip technology to environmental monitoring; in particular, the implementation of an innovative system-on-chip microwave radiometer for temperature remote sensing and its application to fire detection. The quantum theory of radiation says that every body characterized by a temperature higher than zero Kelvin emits electromagnetic radiation. The radiated power can be measured even without physical contact with the body itself by means of a microwave radiometer, which is basically a highly sensitive radio receiver. Indeed, the environment generally includes several objects of different nature such as atmosphere, vegetation, and land or sea surfaces and it is possible to obtain a remarkable amount of information about their conditions by measuring the power they radiate. In this way, burning wood or frozen road pavement can be detected or even the measurement of the power radiated by the atmosphere can provide an estimate of the amount of water vapor or liquid water in the clouds. The characteristics of electromagnetic emission vary 
remarkably with frequency. Gaseous objects, for example, are characterized by spectral lines (bands) corresponding to transitions between different energy levels in atoms or molecules. The resonance frequencies of atmospheric water vapor $(22 \mathrm{GHz}$ and $183 \mathrm{GHz})$ and oxygen $(60 \mathrm{GHz}$ and $118 \mathrm{GHz})$ can be observed within microwave and millimeter-wave bands. Solid and liquid objects, however, are characterized by a radiation spectrum similar to a black body, once a proper frequency-dependent correction factor called "emissivity" is considered. Moreover, vegetation, land, sea surfaces, and ice need to be observed in the frequency bands where the atmosphere is transparent to radio waves.

In the past few years, environmental remote sensing has experienced significant growth thanks to the technological evolution of radiometers both for terrestrial and space applications. At the same time, we have witnessed an ever increasing interest in radiometer networks for atmospheric remote sensing. In fact, due to extensive deployment they should allow for improvement in both meteorological forecasts and climatologic studies. On the other hand, the actual implementation of such a network requires the development of innovative systems characterized by excellent performance, low production costs, low weight and small size, low power consumption, and wireless connectivity. In order to satisfy cost, size, and power consumption requirements, several projects based on monolithic integrated circuit implementation have been developed by using gallium-arsenide (GaAs) or indium-phosphide (InP) technologies since these III-V compound semiconductors are well suited for the fabrication of low-noise, millimeter-wave devices. These systems can reach very low noise performance with efficient calibration mechanisms and compensation techniques against temperature variations (typically implemented by using Peltier's cells). However, these radiometric sensors do not live up to expectations and the result is a bulky and expensive device so that its potential as a sensor is drastically limited to a few applications.

As previously mentioned, the latest generation of standard CMOS silicon processes provide active devices characterized by extremely low noise contributions (e.g. typical noise figure close to $1 \mathrm{~dB}$ at $10 \mathrm{GHz}$ ) with associated low-power consumption. The use of the CMOS technology would allow fabricating the receiver front-end and the intermediate frequency electronics as well as that dedicated to signal processing, system calibration, and functionality testing on a single chip. The System-on-Chip (SoC) approach would have a remarkable and beneficial impact on the design of microwave radiometers. For example, the strategies of thermal stabilization necessary to reduce the drift between successive calibrations, thus achieving the required sensitivity, could be much simpler and more effective if applied to a single-chip receiver. It should also be noted that, since gain stability in the receiver chain is one of the most critical requirements for correct system operation, the use of MOS devices is to be preferred over that of bipolar ones that are more sensitive to changes in environmental conditions. Moreover, the minimum noise figure of MOS transistors, which was previously their main drawback in comparison with bipolar ones, shows a steady decrease in the last few years as the cutoff frequency increases; thus the choice of the CMOS process to implement a new generation of low-cost low-power radiometers. Furthermore, it allows us to realize distributed wireless sensor networks for temperature remote sensing, as in the case of environmental monitoring and civil safeguard.

Here we consider the use of a radiometer to determine the increment of the antenna equivalent noise temperature due to black-body emission from a fire. To do this, a feasibility study of this sensor in a silicon single-chip microwave radiometer has been carried out. This single-chip solution, due to the dramatic reduction of cost, size, and power consumption can also be exploited to build radiometric imaging systems. These systems are constituted by an array of sensors (the resolution of which increases with the operating frequency and with the number of elements of the array) and are employed for medical applications or civil security. For example, this sensor can be used to prevent forest fires or to help firemen detect an unseen fire behind a door or wall.

\subsection{Fire Detection by Microwave Radiometer}

How is a fire detected by a microwave radiometer? What are the different factors measured by the radiometer antenna? This section answers these questions from a theoretical point of view. In order 
to model the scenario sensed by a microwave radiometer the approach described in [28] has been adopted. First, the case of forest fire detection will be considered then the same principles will be applied to fire detection in indoor environments.

\subsubsection{Forest Fires}

In dealing with forest fires, the scene sensed by a microwave radiometer can be described by the scheme in Fig. 14. The brightness temperature of the scene $T_{A P}$ in the direction $(\theta, \varphi)$ is given by

$$
T_{A P}(\theta, \varphi)=T_{U P}(\theta, \varphi)+Y(\theta, \varphi)\left[T_{B}(\theta, \varphi)+T_{S C}(\theta, \varphi)\right]
$$

where $T_{B}(\theta, \varphi)$ is the contribution due to the forest and, eventually, to the fire in it, $T_{U P}(\theta, \varphi)$ is the upward atmospheric temperature at height $h, T_{S C}(\theta, \varphi)$ is the scattered radiometric temperature of the area under observation, and $Y(\theta, \varphi)$ is the atmospheric transmissivity. At low heights, both $T_{U P}$ and $Y$ can be neglected so

$$
T_{A P}(\theta, \phi)=T_{B}(\theta, \varphi)+T_{S C}(\theta, \varphi) .
$$

In particular, $T_{S C}$ is due to the downward atmospheric temperature $T_{D N}$ scattered by the forest vegetation, the latter having a reflectivity $\Gamma_{V}$ defined as

$$
T_{S C}(\theta, \varphi)=\Gamma_{V}(\theta, \varphi) T_{D N}(\theta, \varphi) .
$$

The term $T_{B}$ is composed of many terms, the principal ones being the direct emission from the vegetation canopy (emissivity $e_{V}$, physical temperature $T_{V}$ ) and the direct emission from the earth surface (brightness temperature $T_{E}$ ) attenuated by the vegetation (transmissivity $Y_{V}$ ):

$$
T_{B}(\theta, \varphi)=e_{V}(\theta, \varphi) T_{V}(\theta, \varphi)+Y_{V} T_{E}(\theta, \varphi) .
$$

The term not included in (15) is due to the downward emission from the vegetation canopy reflected by the earth surface and transmitted again across the vegetation. Finally, at the thermodynamic equilibrium the following relationship holds:

$$
\Gamma_{V}(\theta, \varphi)+e_{V}(\theta, \varphi)+Y_{V}(\theta, \varphi)=1 .
$$

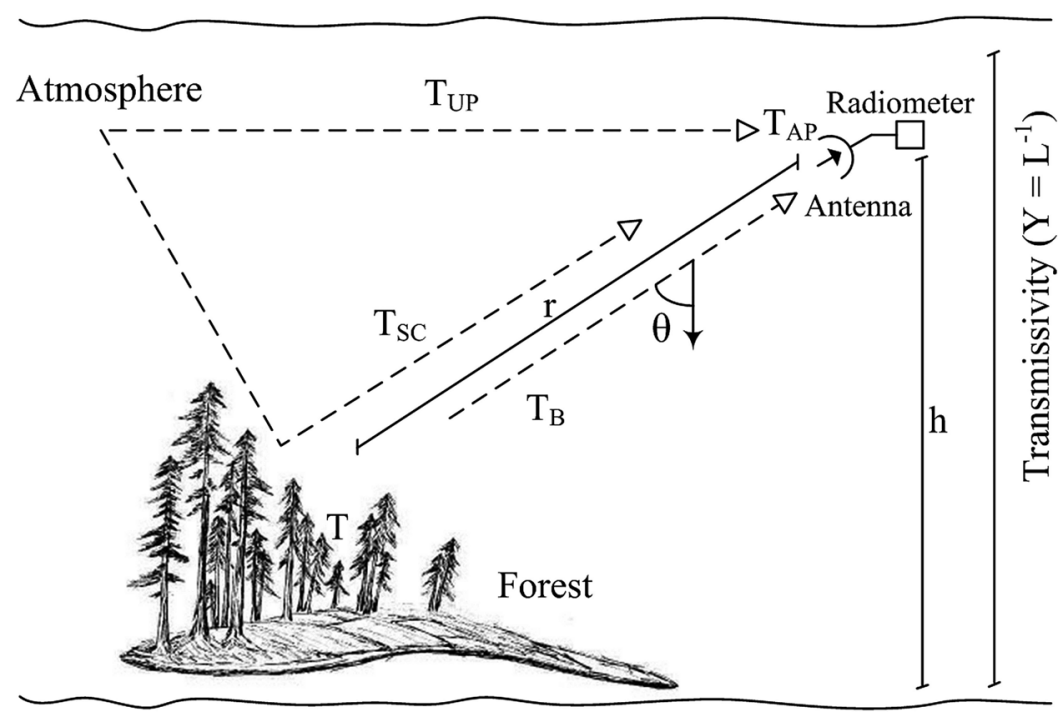

FIGURE 14 Scene sensed by a microwave radiometer in the case of forest fire detection. The fire is masked to an external observer by the vegetation canopy. 
The equivalent antenna noise temperature $T_{A}$, i.e. the noise power received by the antenna itself, can be determined once the brightness temperature of the observed scene is known. Following the definition in [29] such an antenna noise temperature can be written as

$$
T_{A}=\frac{1}{4 \pi} \iint T_{A P}(\theta, \varphi) D(\theta, \varphi) d \Omega
$$

with $D(\theta, \varphi)$ being the antenna directivity. If the antenna beam is sufficiently narrow it will sample the above distributions just in the direction where the antenna is pointed. As a consequence, the integral (17) can be written in an approximate form. Inserting (13)-(15) into (17) one obtains

$$
T_{A}=T_{D N} \Gamma_{V}+e_{V} T_{V}+\frac{Y_{V}}{4 \pi} \iint T_{E}(\theta, \varphi) D(\theta, \varphi) d \Omega .
$$

For the moment, the brightness temperature coming from the earth surfaces has been kept within the integral. This brightness temperature can be modeled with a two-valued function, one value representing the fire within a solid angle $\Omega_{F}$ and another associated with the bare soil on the remaining solid angle $4 \pi-\Omega_{F}$ :

$$
T_{E}=\left\{\begin{array}{ll}
e_{F} T_{F} ; & \Omega \in \Omega_{F} \\
e_{S} T_{S} ; & \Omega \in 4 \pi-\Omega_{F}
\end{array} .\right.
$$

Using (23), the integral in (18) reduces to

$$
\frac{1}{4 \pi} \iint T_{E}(\theta, \varphi) D(\theta, \varphi) d \Omega=e_{F} T_{F} q+e_{S} T_{S}(1-q)
$$

where the antenna theorem has been used (i.e. the integral of the directivity on the whole solid angle is equal to $4 \pi$ ) and $q$ is the filling factor (or coupling efficiency) of the antenna with the fire location, a quantity defined as

$$
q=\frac{1}{4 \pi} \iint D(\theta, \varphi) d \Omega
$$

As a result of the above formulation (24) can be inserted in (18) and the following relationship is obtained, describing what an ideal radiometer will measure in the presence of a forest fire:

$$
T_{A}=T_{D N} \Gamma_{V}+e_{V} T_{V}+Y_{V}\left[e_{F} T_{F} q+e_{S} T_{S}(1-q)\right] .
$$

The antenna noise temperature is due to the sum of many different contributions. To predict the capability of radiometric systems to detect fires, the brightness temperature must be estimated also in the case where the fire is not present. For this purpose one can use (26) with $q=0$ :

$$
T_{A}^{0}=T_{D N} \Gamma_{V}+e_{V} T_{V}+Y_{V} e_{S} T_{S} .
$$

Therefore, the brightness temperature difference in the presence of fire with respect to the standard background, i.e. the radiometric contrast $\rho_{T}$, is given by

$$
\rho_{T}=T_{A}-T_{A}^{0}=Y_{V}\left(e_{F} T_{F}-e_{S} T_{S}\right) q
$$

which means that the radiometric contrast is obtained by weighting the temperature increase due to the fire by the filling factor. Since in actual situations $q$ is well below $1 \%$, a contrast of only a few Kelvins is obtained even for fire temperatures of $1000 \mathrm{~K}$. The radiometer will basically measure the soil temperature (around $280 \mathrm{~K}$ during the summer if observations are made in vertical polarization) such that an instrument resolution of at least $0.5 \mathrm{~K}$ is needed to detect a fire in the early stage.

\subsubsection{Fire Detection in Indoor Environments}

The theory developed above applies well also in the case of indoor fire detection. Some modifications are needed, however, to deal with the geometry of the encountered obstacles. The most typical obstacles encountered in indoor environments are walls and doors. All these obstacles are vertical 
with respect to the ground plane, are characterized by a thickness in the range of 5-30 cm, and are fabricated with a number of different materials such as concrete, bricks, plasterboard, wood, etc. From a radiometric point of view, a wall-like obstacle possesses an emissivity $e_{W}$, a transmissivity $Y_{W}$, a reflectivity $\Gamma_{W}$, and a physical temperature $T_{W}$. Sensing the scene with an antenna at close distance from the wall is to exploit the look-through-wall capabilities of microwaves. In this case the wall is assumed to completely fill the antenna beam, thus resulting in a unity filling factor. As a consequence of such an assumption (26) becomes

$$
T_{A}=e_{W} T_{W}+\Gamma_{W} T_{M}+Y_{W}\left[e_{F} T_{F} q+\left(e_{S} T_{S}+\Gamma_{S} T_{D N}\right)(1-q)\right] .
$$

In this expression $T_{D N}$ represents the microwave emission of the ceiling and $T_{M}$ is the contribution due to sky and soil from the radiometer side. It is useful to note that the soil reflectivity $\Gamma_{S}$ can be related to the soil emissivity by

$$
\Gamma_{S}=1-e_{S}
$$

The wall transmissivity is the most important parameter for indoor fire detection systems. To improve the wall penetration capability a low operating frequency (on the order of a few $\mathrm{GHz}$ ) is preferred. The drawback of this selection is the reduction in spatial resolution for a given antenna size.

\subsection{System-on-Chip Radiometer for Forest Fire Detection}

There are different architectures that allow us to implement a microwave radiometer as: (1) a Dicke radiometer, (2) a noise-injection radiometer and (3) a total power radiometer [30]. The total power architecture has been chosen since it is characterized by both circuit simplicity and excellent sensitivity. The block diagram of the overall system is shown in Fig. 15. The receiver consists of a switch and noise source for the calibration procedure, a low noise amplifier (LNA), a mixer, an amplifier at intermediate frequency (IF), a detector, an integrator, and the Data Acquisition \& Process Unit block which provides the data collected by the sensor for the end-user and carries out the calibration procedure through appropriate control signals (switch driver signal).

For an SoC radiometer microstrip technology is a good candidate to design the antenna because it offers several advantages such as low cost, low profile, and minimum weight. It also allows for

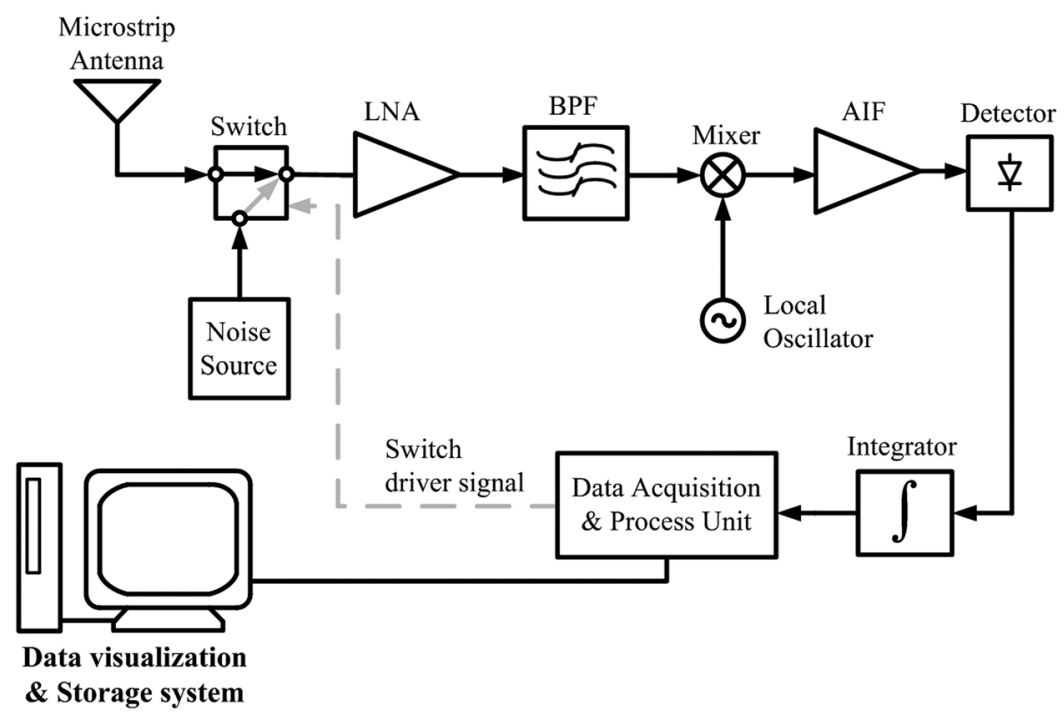

FIGURE 15 Radiometer on silicon for the forest fire prevention: block diagram. 
good directivity when long-range, high spatial resolution detection is sought. Therefore, patch array antennas are interesting solutions for radiometric applications and they are being investigated for a number of Ku-band systems.

The antenna switch for system calibration controls the input into the LNA. The standard implementation consists of a low impedance path in the desired direction and a high impedance path in the other. The insertion loss of the antenna switch is one of the most significant limitations on the sensitivity of the receiver. In RF circuits this operation is typically realized with LC resonant filters to realize both low (series resonant) and high (parallel resonant) impedances. Unfortunately, the low quality factor of integrated LC filters (which is most often limited by the integrated inductor) represents a strong limitation to realize ideal resonant filters. For this reason most widespread commercial solutions consist of bulky expensive components that are external to the chip such as MEMS (MicroElectro-Mechanical Systems), PHEMT (Pseudomorphic High Electron Mobility Transistors), PIN diodes or MESFET (Metal Semiconductor Field Effect Transistor) devices. It has been only recently that fully integrated solutions have been realized on standard microelectronic technologies (CMOS). Their main drawbacks are a non-negligible insertion loss around 1-2 dB and limited maximum operating frequency. A relatively efficient solution is to use the active circuit named the 'Boot-Strapped Inductor' [31] (BSI) which has a demonstrated capability to provide a behavior equivalent to that of high quality factor $\mathrm{Q}$ inductors. Relative to previous approaches, this solution can be realized at higher operating frequencies and with an extremely lower insertion loss (up to $0.1 \mathrm{~dB}$ ).

Low-noise amplifiers (LNAs) are key components in microwave receivers since they allow one to reduce the minimum noise figure (NFmin) of the system, thus maximizing its sensitivity. The NFmin decreases in MOS transistors as the channel length is made shorter and the transconductance (gm) increases. According to literature, a $90 \mathrm{~nm}$ technology can yield NFmin $=0.5 \mathrm{~dB}$ at $5 \mathrm{GHz}$ (with $19 \mathrm{~dB}$ associated gain) and it is lower than $2 \mathrm{~dB}$ between 2 and $18 \mathrm{GHz}$. A $80 \mathrm{~nm}$ process can yield NFmin $=1.1 \mathrm{~dB}$ at $18 \mathrm{GHz}$ and $13 \mathrm{~dB}$ associated gain. These values are quite close to those offered by SiGe HBTs and support the choice of a $90 \mathrm{~nm}$ CMOS technology for this project.

The second stage of the receiver is the mixer that down-converts to intermediate frequency the LNA output signal. Although the noise figure requirements are not stringent, they nevertheless have to be accounted for and evaluated in relationship to those of the LNA and its gain in such a way as to make the overall system noise figure approximately equal to that of the LNA. Per the literature, a standard $180 \mathrm{~nm}$ CMOS technology can yield mixer noise figures of $13 \mathrm{~dB}$ at $13 \mathrm{GHz}$ and $18 \mathrm{~dB}$ at $24 \mathrm{GHz}$.

The local oscillator is another key component in the definition of the system specifications. The Phase-locked Loop (PLL) solution is the only one allowing for the required accuracy and stability of the oscillation frequency. The large demand for RF integrated circuits working in $\mathrm{C}, \mathrm{X}$ and $\mathrm{Ku}-$ bands for wireless applications and optical telecommunications can be combined with the rapid development of CMOS technologies. In particular, a critical parameter of the voltage controlled oscillator (VCO) is phase noise which severely impacts the performance of the whole receiver due to inter-modulation and noise folding. The goal of integrating a whole high-frequency PLL requires designing a $\mathrm{VCO}$ with a tuning range wide enough to compensate for the parametric variations of active and passive components, which can be significant. The literature indicates that the $90 \mathrm{~nm}$ CMOS technology certainly has the potential for delivering a $13 \mathrm{GHz}$ PLL with satisfactory phasenoise, stability, and power dissipation performance.

For radiometric applications, a relatively large frequency band on the order of a few hundreds of $\mathrm{MHz}$ is required to receive sufficient power. An architectural solution such as a Low-IF allows integrating the IF filter in the same chip as the rest of the receiver. The intermediate frequency amplifier (AIF) must therefore be followed by a low-pass filter and it can be effectively implemented using Gm-C cells. This kind of solution allows for digital control of the cut-off frequency, which is directly linked with the sensitivity of the detector. Finally, as far as the quadratic detector is concerned, it is possible to exploit the quadratic non-linearity offered by MOS transistors. 


\subsection{System Analysis}

The first step in order to implement the microwave radiometric sensor is to define the operating range and a possible scenario of application. As for the operating frequency, it is worth mentioning that the frequencies of interest span from C-band $(4-6 \mathrm{GHz})$ to X-band $(8-12 \mathrm{GHz})$ for fires and biomass monitoring, while experiments at $10 \mathrm{GHz}$ and $37 \mathrm{GHz}$ are helpful in the case a frozen road pavement. Moreover, the water (either steam or liquid) content of the atmosphere can be observed at $23.8 \mathrm{GHz}$ and $31.4 \mathrm{GHz}$, sometimes in conjunction with a $13 \mathrm{GHz}$ channel.

A possible scenario of interest for a $13 \mathrm{GHz}$ microwave radiometer for forest fire prevention is that highlighted in Fig. 14. A first-order theoretical analysis has allowed us to carry out a preliminary design of the overall radiometer and define the specifications of all the building blocks in accordance with the characteristic performance attainable with an implementation on a standard CMOS $90 \mathrm{~nm}$ process (including the supply voltage and the maximum available power from each stage). This analysis has allowed us to define the sensitivity of the radiometer. In particular, the sensitivity of a total power radiometer $(\Delta T)$, to a first approximation, is given by

$$
\Delta T=\frac{T_{A}+T_{N}}{\sqrt{B \tau}}
$$

where $T_{A}$ is the equivalent temperature of the antenna, $T_{N}$ is the equivalent noise temperature of the receiver, $\tau$ is the integration time, and $B$ is the bandwidth of the receiver. Moreover, it is worth nothing that the channel transmissivity is approximately equal to 1, as shown in Fig. 16, over the whole band of interest (12.9-13.1 GHz; $200 \mathrm{MHz}$ within the ISM band) so that atmospheric attenuation can be neglected.

A band of interest equal to $200 \mathrm{MHz}$ around $13 \mathrm{GHz}$ and a temperature range of the receiver between 220 and $1100 \mathrm{~K}$ have been considered. This range of temperature allows us to estimate the output power from each block and the output voltage of the receiver (i.e. the integrator output voltage) when an input power between the highest and lowest input power of the receiver, respectively, is given. However, it is worth mentioning that this range corresponds to the temperature interval of interest for this application. In particular, $200 \mathrm{~K}$ is the minimum temperature for a forested region on the Earth surface, whereas $1100 \mathrm{~K}$ is the highest combustion temperature that a forest fire could develop. The radiometer will measure such a temperature only observing the fire with a unity filling factor $(q=1)$. Then, we assumed a distance $r$ of $200 \mathrm{~m}$ between the sensor and the forest area under

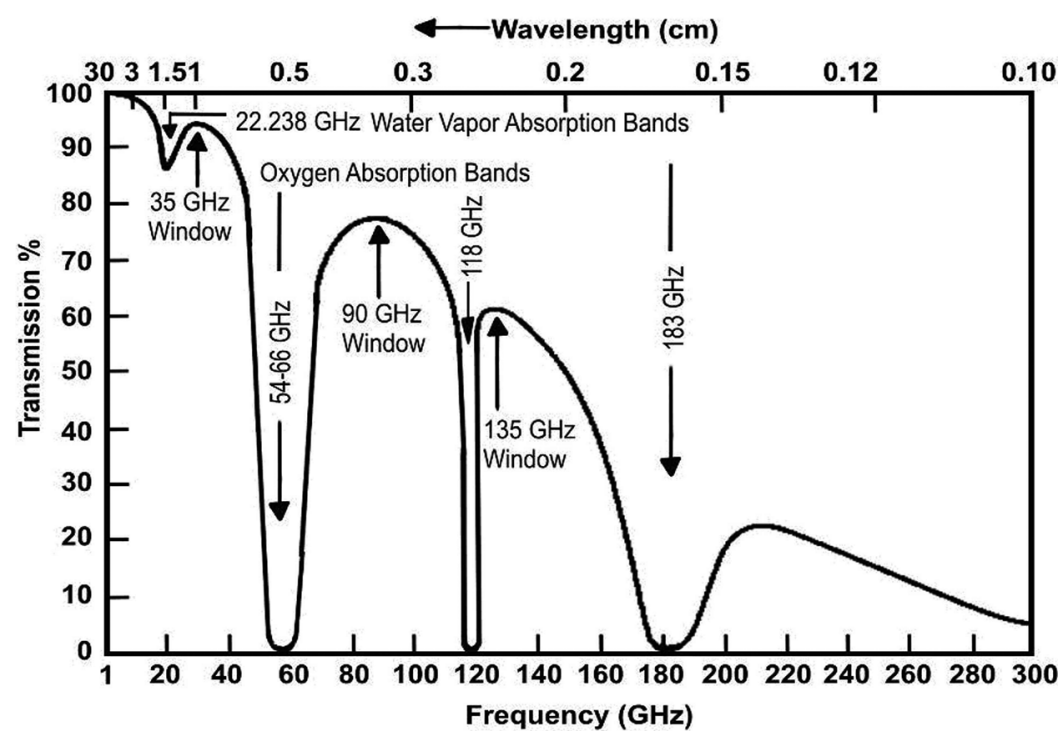

FIGURE 16 Atmospheric Trasmissivity vs. frequency and wavelength. 
TABLE 2 Radiometer Specifications

\begin{tabular}{cclc}
\hline$\Delta T$ & $0.2 \mathrm{~K}$ & Antenna Gain & $17 \mathrm{dBi}$ \\
$r$ & $200 \mathrm{~m}$ & zenithal/ azimuthal & $6^{\circ} / 40^{\circ}$ \\
$N F$ & $4.85 \mathrm{~dB}$ & Range Temp. & $220-1100 \mathrm{~K}$ \\
\hline
\end{tabular}

TABLE 3 Specifications of the Building Blocks

\begin{tabular}{lllll}
\hline & Switch & LNA & Mixer & AIF \\
\hline Noise Figure & $\leq 1 \mathrm{~dB}$ & $\leq 3 \mathrm{~dB}$ & $\leq 15 \mathrm{~dB}$ & $\leq 10 \mathrm{~dB}$ \\
Power Gain & & $\geq 20 \mathrm{~dB}$ & $\geq 4 \mathrm{~dB}$ & $\geq 35 \mathrm{~dB}$ \\
\hline
\end{tabular}

observation, a real microstrip patch antenna with a gain of $17 \mathrm{dBi}$, and zenithal and azimuthal angles of $6^{\circ}$ and $40^{\circ}$, respectively (the sensor is mounted on the same microstrip board of the antenna). In this way, the area observed by the radiometer amounts to $2260 \mathrm{~m}^{2}$. Finally, if we assume the temperature range as mentioned above, a maximum power at the input of the detector between -30 and $-25 \mathrm{dBm}$, a detector sensitivity of $10 \mathrm{mV} / \mu \mathrm{W}$ and an integration time of about $70 \mathrm{~ms}$, then the radiometric sensitivity $(\Delta T)$ required for its application to forest fire detection is $0.2 \mathrm{~K}$ and the equivalent noise temperature of the receiver $\left(T_{N}\right)$ amounts to $596 \mathrm{~K}$ corresponding to a noise figure of $4.85 \mathrm{~dB}$. The specifications of the $\mathrm{SoC}$ microwave radiometer are reported in Table 2 and the specifications of each building block are summarized in Table 3.

\subsection{CAD System Analysis}

The results obtained by the first-order theoretical analysis have been confirmed by an EDA CAD tool analysis. The overall radiometer has been implemented by functional blocks in the CAD tool environment, including several non-ideal effects such as: (a) physical temperature of the antenna, (b) equivalent noise contributions, and (c) bandwidth and linearity performance limitations introduced by each building block if realized on the CMOS $90 \mathrm{~nm}$ silicon process. It is worth nothing that this pure-CMOS solution is extremely attractive since the results of this project are widely transferable to a large number of diverse applications. This is the rationale for choosing a last-generation CMOS technology (the $90 \mathrm{~nm}$ CMOS process by ST-Microelectronics).

The CAD system analyses have been performed by means of the Ptolemy simulator within the software environment Advanced Design System (ADS2005A) by Agilent Technologies. In particular, the first simulations have been carried out by using the library model provided by the software environment in order to confirm the results obtained by the theoretical analysis. Then, the building blocks have been designed in CMOS $90 \mathrm{~nm}$ process by ST-Microelectronics by using the software environment Cadence ${ }^{\mathrm{TM}}$ and the simulation results have been introduced as black boxes (instead of the library models) in the Ptolemy simulator in order to estimate the influence of each block on the global performance of the radiometer. This procedure has allowed us to refine the design on the most critical blocks (such as the LNA).

Simulations with different equivalent antenna noise temperatures have been carried out. To mimic a signal of interest, a block emulating a white thermal noise with Gaussian probability density has been implemented and included as a source in the simulation scheme. In particular, simulations have been performed with equivalent noise temperatures $\left(T_{A}\right)$ between $55 \mathrm{~K}$ and $152 \mathrm{~K}$ (corresponding to a range of temperature of the area under observation between $220-1100 \mathrm{~K}$ ) and with a difference of $0.2 \mathrm{~K}$ (the radiometric sensitivity of our receiver). These simulations have confirmed that the receiver is capable ideally of reaching such a radiometric resolution. In particular, the output voltages of the receiver, obtained by theoretical and CAD tool analyses, are reported in Table 4 when the equivalent noise temperatures are: (i) $T_{A, \max }=152 \mathrm{~K}$, (ii) $T_{A, \min }=55 \mathrm{~K}$, (iii) $\Delta T_{A, \max }=\left(T_{A, \max }-T_{A, \min }\right)=97 \mathrm{~K}$ and (iv) $\Delta T_{A, \min }=0.2 \mathrm{~K}$, respectively. Note the good agreement between the results obtained 
TABLE 4 Output Voltages of the Receiver

\begin{tabular}{ccc}
\hline Output Voltage & Theoretical results $(\mathbf{m V})$ & CAD results $(\mathbf{m V})$ \\
\hline$V_{M A X}$ & 12.892 & 12.444 \\
$V_{M I N}$ & 11.203 & 11.414 \\
$\Delta V_{M A X}$ & 1.689 & 1.03 \\
$\Delta V_{M I N}$ & $3.48 \times 10^{-3}$ & $2.86 \times 10^{-3}$ \\
\hline
\end{tabular}

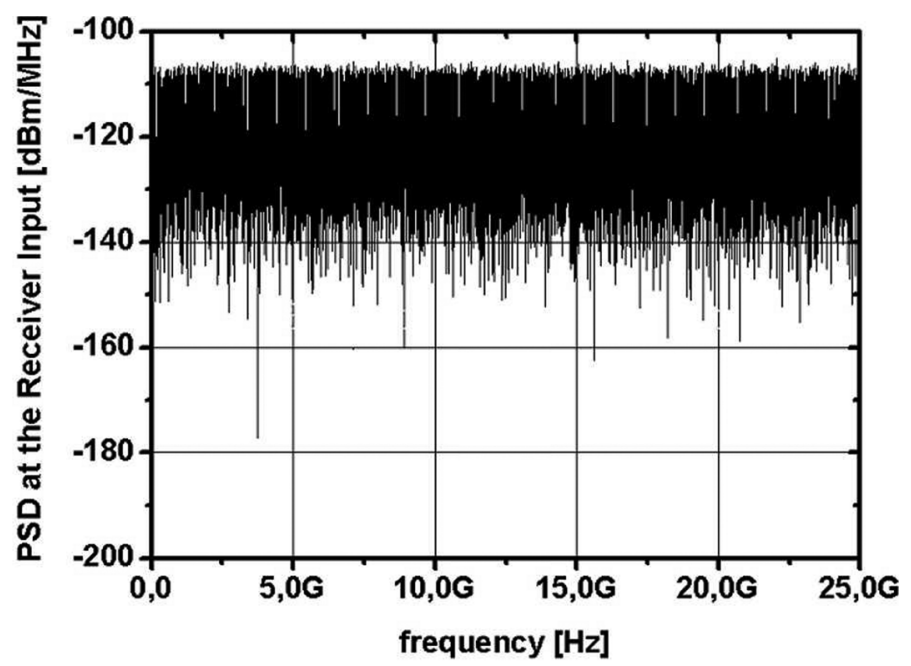

FIGURE 17 Power Spectral Density (PSD) at the input of the receiver.

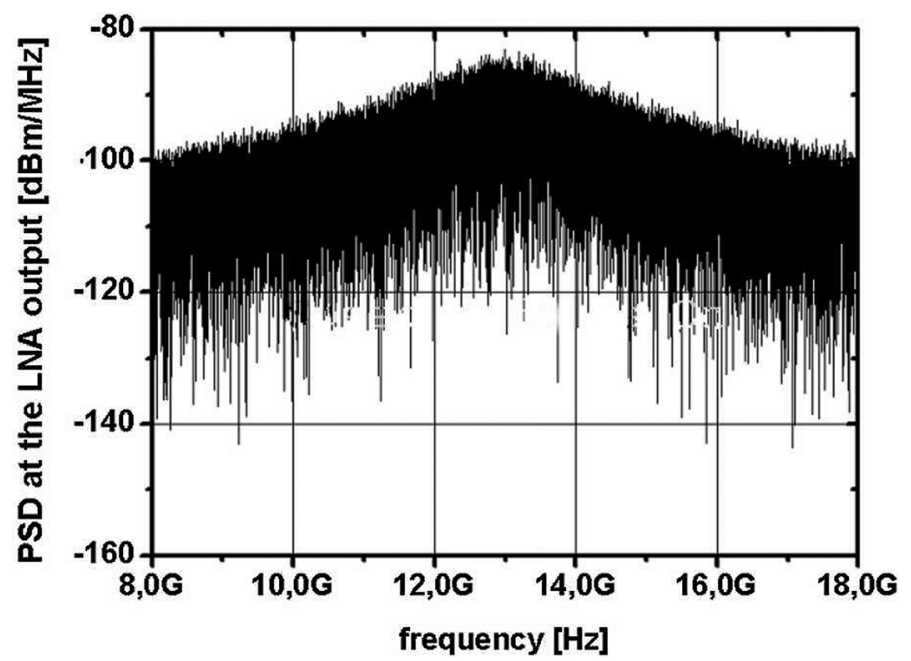

FIGURE 18 Power spectral density at the output of the Low Noise Amplifier (LNA).

with theoretical analysis and CAD simulation results. Moreover, the representative power spectral densities (PSD) of the power at the input of the receiver and at the output of each block obtained by simulations are reported in Figs. 17-20. The output voltages (at the output of the integrator) obtained for the highest and lowest power levels collected by the antenna are reported in Fig. 21. 


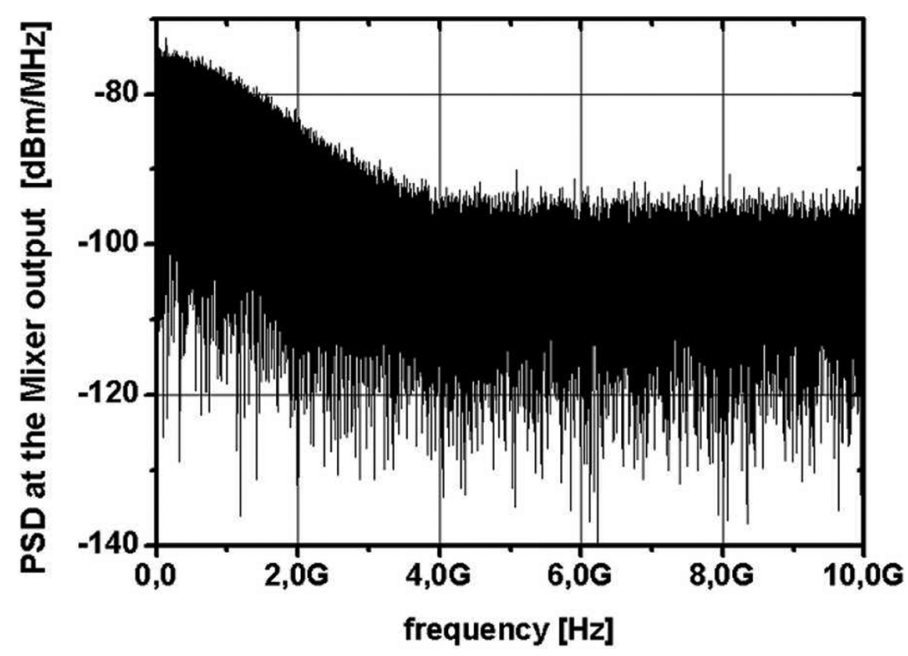

FIGURE 19 Power spectral density at the output of the Mixer.

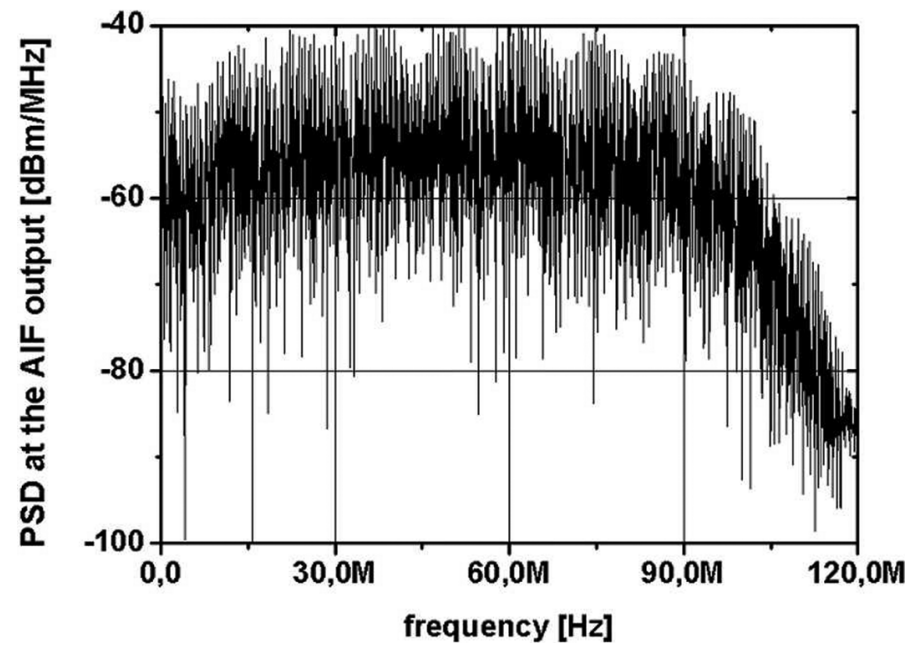

FIGURE 20 Power spectral density at the output of the Amplifier at the Intermediate Frequency (AIF).

\subsection{System Validation through a Hybrid Prototype}

To confirm experimentally that microwave radiometers are able to detect fires masked by obstacles of various nature, in both outdoor and indoor environments, a proof-of-the-concept has been realized. To achieve this, a discrete-component $12.65 \mathrm{GHz}$ radiometer has been used.

The discrete-component $12.65 \mathrm{GHz}$ radiometer was described in detail in [32]. For the reader's clarity its design and operation will only be recalled here. The receiver consists of a Low Noise Block (LNB) for Satellite Television (SAT-TV) applications which is characterized by low-cost and high performance. In particular the LNB features a noise figure of $0.8 \mathrm{~dB}$, an RF-gain of $54 \mathrm{~dB}$, and a gain drift versus temperature lower than $0.1 \mathrm{~dB} / \mathrm{K}$. The LNB converts the RF-signal between $11.65 \mathrm{GHz}$ and $12.75 \mathrm{GHz}$ into an IF-signal in the range $0.95 \mathrm{GHz}$ to $2.05 \mathrm{GHz}$. The image frequency rejection is more than $50 \mathrm{~dB}$ by means of a microwave band-pass filter integrated into the LNB. The IF chain provides a $10 \mathrm{~dB}$ gain over a bandwidth of $100 \mathrm{MHz}$. The IF filter has a center frequency of $1.95 \mathrm{GHz}$. The assembly of the sensor is illustrated in Fig. 22. 


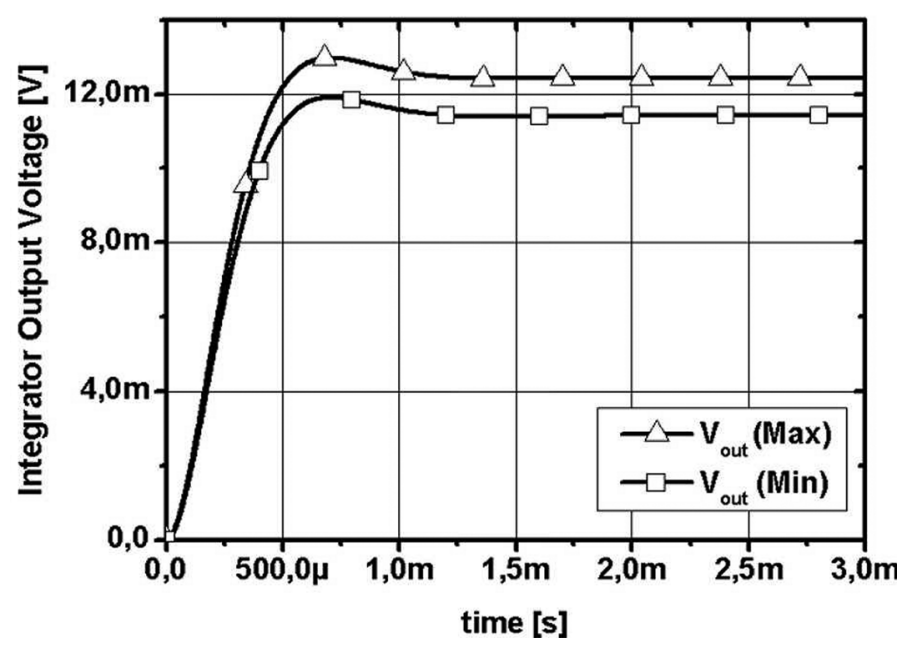

FIGURE 21 Receiver output voltage where $V_{O U T, \text { max }}$ and $V_{O U T, \text { min }}$ are the voltages at the output of the integrator with $T_{A}=T_{A, \max }$ and $T_{A}=T_{A, \min }$, respectively.

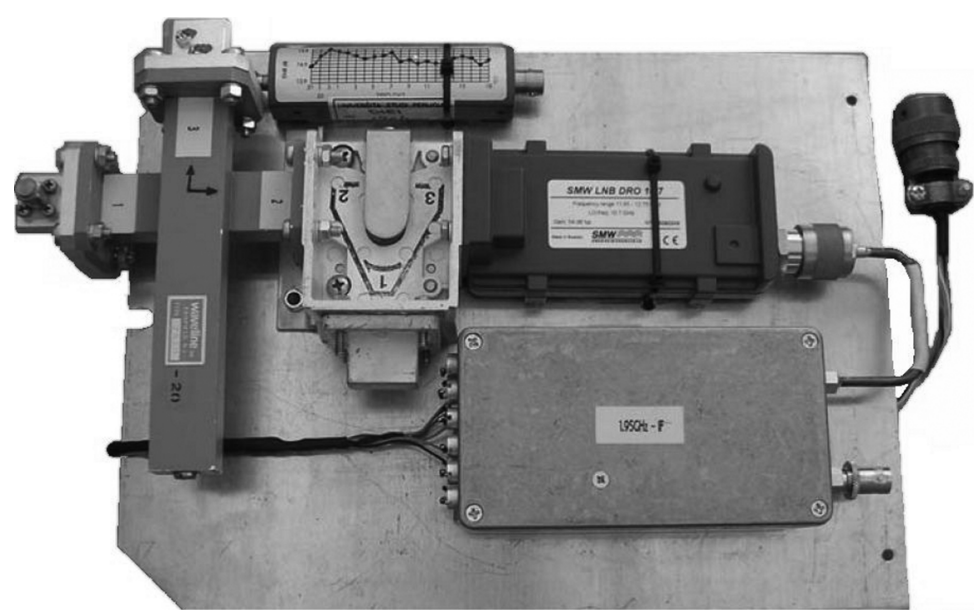

FIGURE 22 Assembly of the $12.65 \mathrm{GHz}$ discrete-component radiometer. From the left: input waveguide, $20 \mathrm{~dB}$ Moreno coupler, isolator, SAT-TV low-noise down-converter, calibration noise source on the top, and $1.95 \mathrm{GHz}$ IF chain on the bottom.

The antenna noise temperature $\left(T_{A}\right)$ measured by the sensor can be obtained according to the noise-adding equation [33]

$$
T_{A}=\alpha \cdot \frac{V_{O F F}}{V_{O N}-V_{O F F}}-\beta
$$

where $V_{O N}$ and $V_{O F F}$ are the output voltage obtained when the noise source is switched on/off, respectively, while $\alpha$ and $\beta$ are the scale factor and the offset of the radiometer, respectively. These constants have been determined by suitable calibration procedures, the best estimation of which being: $\alpha=87.4 \mathrm{~K}, \beta=113.0 \mathrm{~K}$. It is worth noting that such an instrument can run without thermal stabilization producing an absolute error less than $4 \mathrm{~K}$; this if the physical temperature of the receiver is in the range $3-23^{\circ} \mathrm{C}$. 


\subsubsection{Outdoor Experiments}

Field experiments were carried out in a rural area of central Italy during Spring 2007 . A $60 \mathrm{~cm}$ offset dish antenna was used during these experiments. This antenna was characterized by a half-power beamwidth of $3^{\circ}$ and a gain of $37 \mathrm{dBi}$. The scene was sensed in horizontal $(\mathrm{H})$ polarization with an incidence angle of $84^{\circ}$. The observation distance was about $30 \mathrm{~m}$. Consequently, the antenna footprint was approximately equal to $19 \mathrm{~m}^{2}$. The test fire was realized with oak and had an area of about $0.38 \mathrm{~m}^{2}$. The soil temperature was, on average, close to $300 \mathrm{~K}$ while the soil emissivity can be in the range $0.5-1$, depending on the moisture content of the soil itself, observation angle, and polarization. The fire temperature can easily reach $1000 \mathrm{~K}$ (red embers). Since, in actual situations, the filling factor is well below $1 \%$, the expected contrast is of only a few Kelvins. The filling factor obtained in this case is about $2 \%$.

Figure 23 shows the measured antenna noise temperature. The receiver is calibrated at its input terminals but neither antenna efficiency nor feed return loss have been compensated (i.e. the raw data are reported in the figure). The average air temperature was $292 \mathrm{~K}$, whereas an apparent soil temperature around $200 \mathrm{~K}$ is obtained. This can be explained considering a soil emissivity around 0.6-0.7 for horizontal polarization observations at high incidence angles. During the outdoor experiment the radiometric contrast was measured by covering the fire with a metallic screen: a value of $8 \mathrm{~K}$ is so obtained (interval $A$ of Fig. 23), clearly detected by the sensor.

\subsubsection{Indoor Experiments}

The indoor experiments have been carried-out with the purpose to detect a fire masked by a wall or a door. This is a scenario of great interest for the safety of firemen. The experimental set-up is shown in Fig. 24 where the soil profile is constituted by the soil itself and the furnace. All the experiments have been carried out with the fire size and material kept constant. The fire was been produced by 21 pine sticks forming a pile of $0.25 \mathrm{~m}$ on a side. The total mass of the wood is $1.18 \mathrm{~kg}$ while the area of the fire is: $A_{\text {FIRE }}=0.0625 \mathrm{~m}^{2}$. Finally, the estimated filling factor is $q=3.2 \%$.

The measured results are reported in Fig. 25. In particular, such a figure shows a reference experiment with no wall, another experiment with a light plasterboard wall, and a final test with a

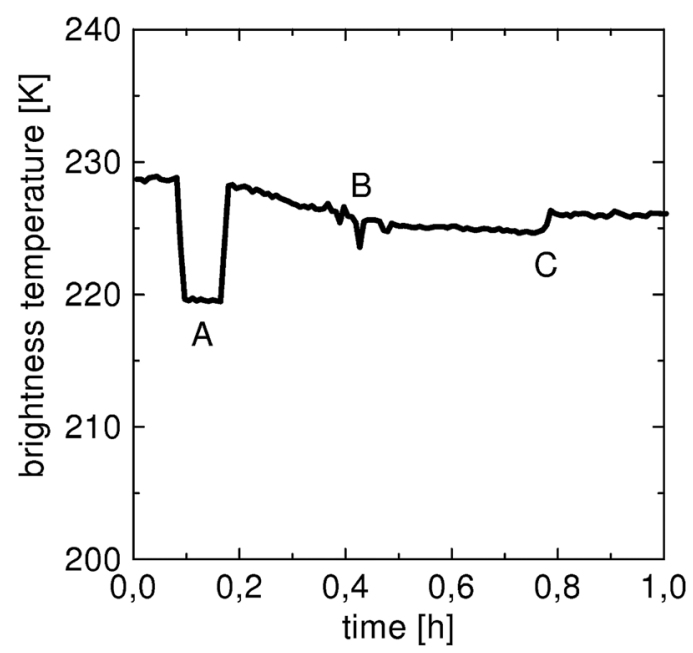

FIGURE 23 Antenna noise temperature of the outdoor experiment: the radiometric contrast of $8 \mathrm{~K}$ (interval A) is obtained covering the fire with a metallic screen. The spikes in B are caused by the authors crossing the antenna beam. Step $\mathrm{C}$ is due to an abrupt change of the fire geometry: a stick of wood of about $7 \mathrm{~cm}$ diameter is broken by the fire thus exposing the embers underneath. 


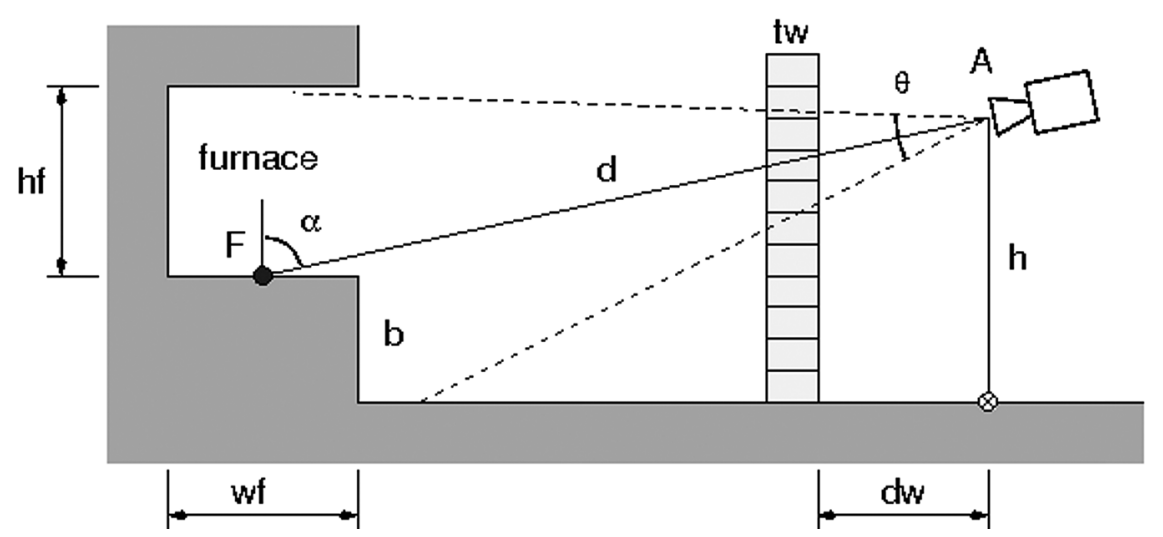

FIGURE 24 Basic set-up used for the inter-wall fire detection experiments. The geometry is characterized by: $d=2.40 \mathrm{~m}, h=0.49 \mathrm{~m}, w f=0.6 \mathrm{~m}, d w=0.52 \mathrm{~m}, t w=0.125 \mathrm{~m}, h f=0.64 \mathrm{~m}, b=0.41 \mathrm{~m}, \theta=30^{\circ}, \alpha=78^{\circ}$. $\mathrm{A}$ is the antenna and $\mathrm{F}$ is the fire.

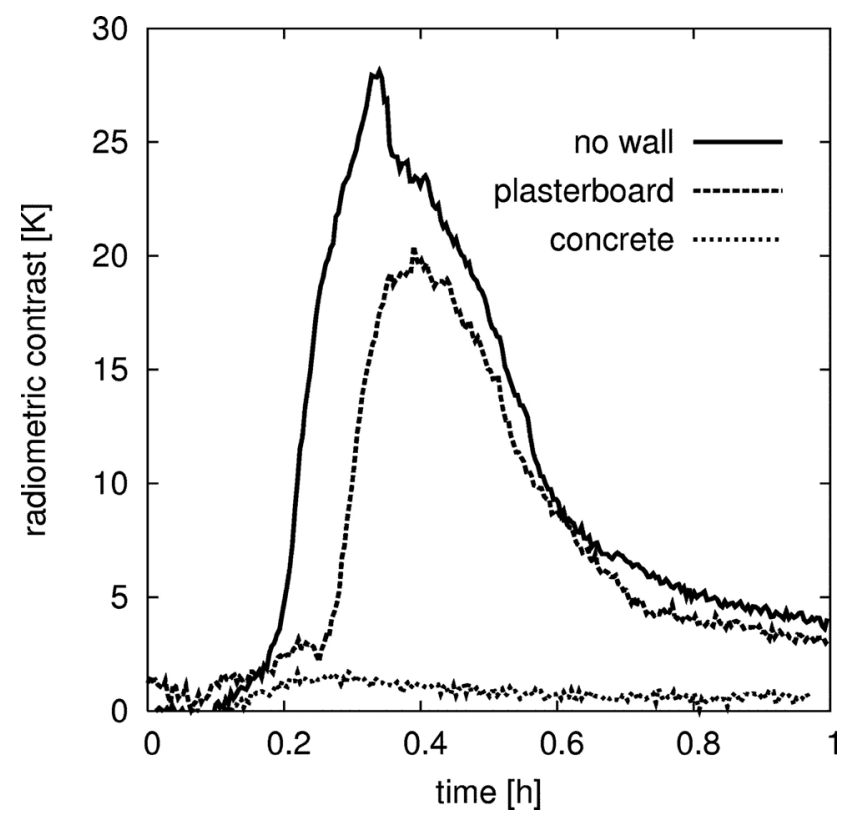

FIGURE 25 Radiometric contrast measured during the inter-wall fire detection experiments. The results without the wall are compared with that of plasterboard and heavy concrete walls. Although the heavy concrete wall causes a significant attenuation, the microwave emission is clearly captured with a radiometric contrast of about $1.4 \mathrm{~K}$.

heavy concrete wall. The radiometric contrast is computed as the antenna temperature difference between the values measured at each time instant minus the values recorded at the beginning of the experiment, i.e. when no fire is present. In particular, using the radiometric contrast $\rho_{T}=27 \mathrm{~K}$ obtained without the wall, the term $\epsilon_{F} T_{F}$ has been estimated to be around $1100 \mathrm{~K}$. By inserting the plasterboard wall a radiometric contrast $\rho_{T}=17 \mathrm{~K}$ is measured, leading to $Y_{W}=0.63$. Finally, in the case of the heavy concrete wall, the contrast amounts to only $1.4 \mathrm{~K}$ and $Y_{W}$ drops to 0.05 . 
TABLE 5 Walls Parameters

\begin{tabular}{lccccc}
\hline Material & Average Density $\left[\mathrm{kg} / \mathbf{m}^{3}\right]$ & $\boldsymbol{t}_{W}[\mathbf{m}]$ & $\boldsymbol{d}_{W}[\mathbf{m}]$ & $\rho_{T}[\mathbf{K}]$ & $\boldsymbol{Y}_{W}$ \\
\hline Plaster-board & 112 & 0.125 & 0.52 & 17 & 0.63 \\
Concrete & 2063 & 0.125 & 0.52 & 1.4 & 0.05 \\
\hline
\end{tabular}

It is worth noting that the above transmissivity values are correlated with the density of the wall material, as reported in Table 5. Moreover, in spite of a concrete wall introducing a significant attenuation, the microwave emission peak is clearly captured with a radiometric contrast of about $1.4 \mathrm{~K}$, whereas typical IR sensors fail even behind the plasterboard wall.

In conclusion, this proof-of-concept has shown that a microwave radiometer can easily detect a small fire. Furthermore, this detection is possible even if the fire is masked by non-transparent obstacles such as building walls. The discrete-component system has a cost of about $\$ 1800$ and a power consumption of around $3 \mathrm{~W}$. To obtain a further reduction in cost, size, and power consumption the SoC solution proposed in the next section is under development.

\subsection{Present Status for the SoC Radiometer}

At present (i.e. Q3/2008), each building block of the overall radiometer has been designed and realized in a 90-nm CMOS standard process. The details regarding the circuit implementation can be found in [34]. Most of the building blocks (Low Noise Amplifier, Intermediate Frequency Amplifier, Square Detector, Local Oscillator) have been tested successfully. As for the Mixer, the experimental characterization is in progress and the overall system-on-chip integration is scheduled for the Q1/2009. For reasons of space we report hereinafter only the micrographs of the LNA and square detector test-chips (see Figs. 26 and 27), and their most relevant performance (see Figs. 28 and 29).

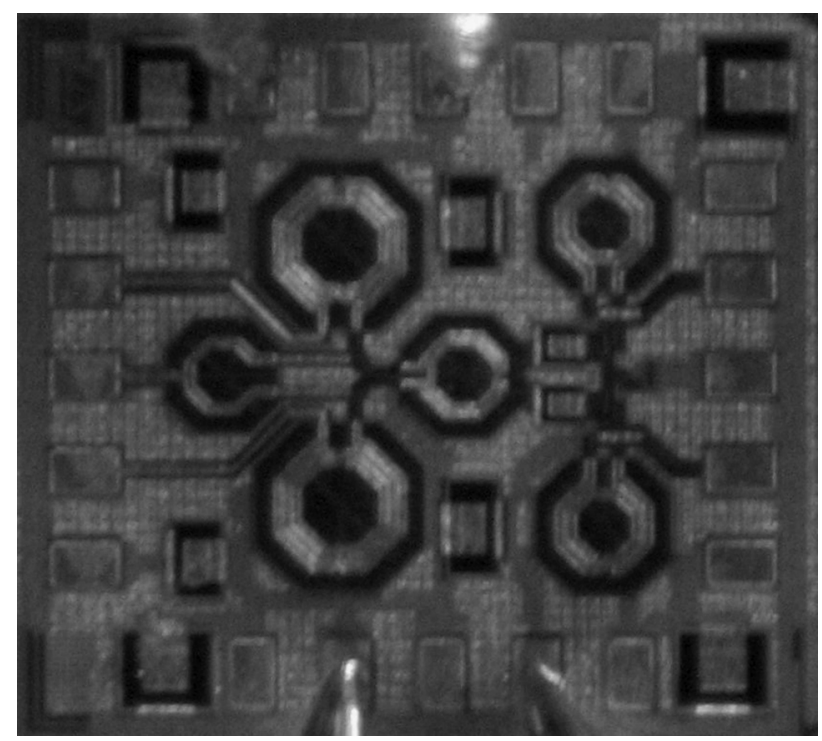

FIGURE 26 LNA testchip. The area size on die amounts to $0.51 \mathrm{~mm}^{2}$ (pads inclusive). 


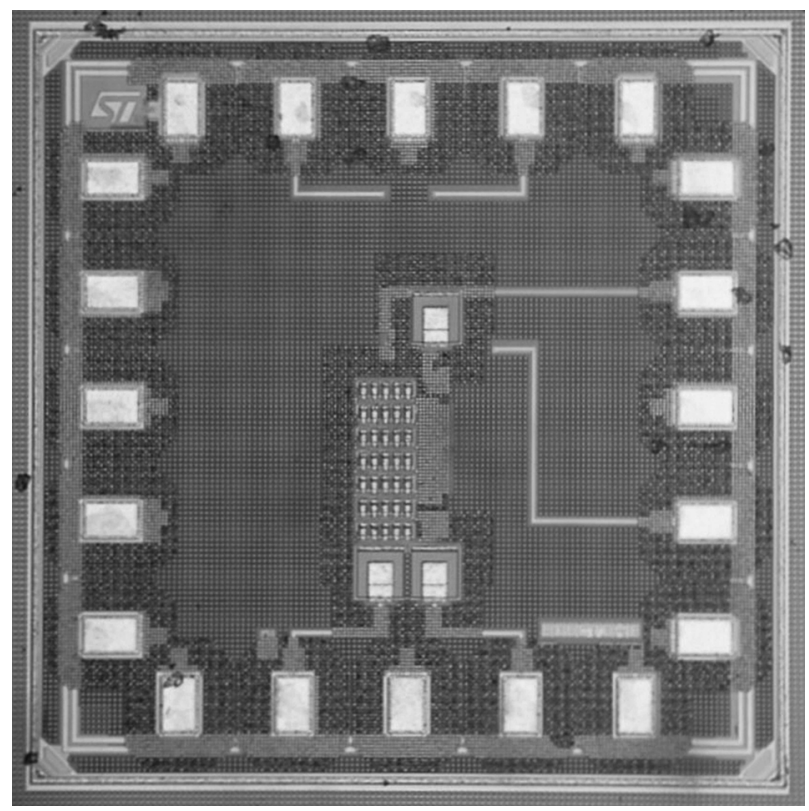

FIGURE 27 IF amplifier, IF filter and square-law detector testchip. The area size on die amounts to $1 \mathrm{~mm}^{2}$ (pads inclusive).

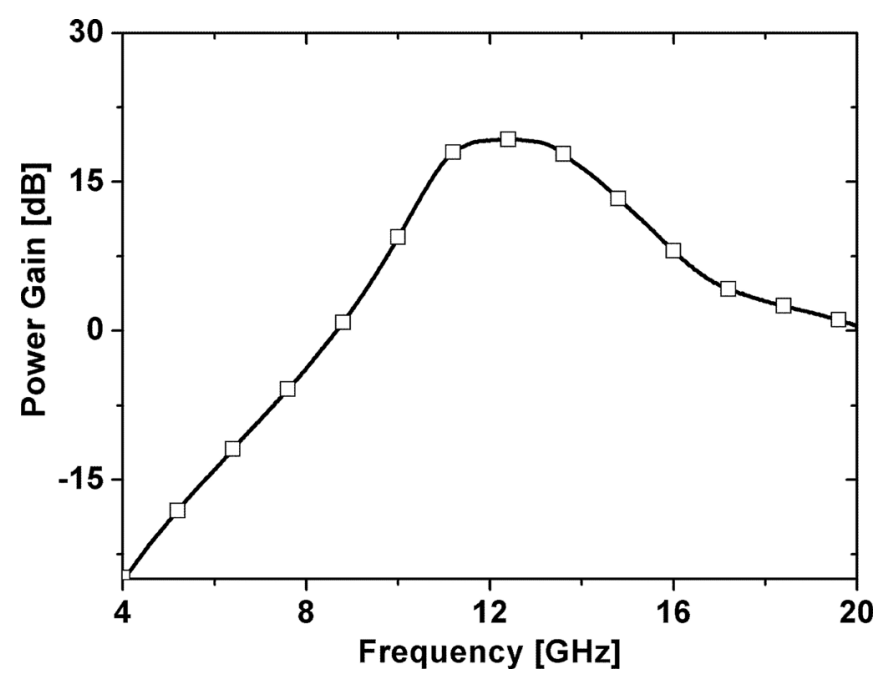

FIGURE 28 LNA power gain vs. frequency (measured).

\section{Conclusions and Future Work}

Despite the fact that the system-on-chip microwave radiometer integration has been targeted for the case of forest fire detection, it is obviously not limited to this specific application. In fact, it is worth mentioning that such a system-on-chip perspective, characterized by miniaturization and low-cost mass-market production, enables its application to an array-based approach (i.e. sensor array) and wearable systems. As for the sensor array, some of the attractive applications are high-resolution imaging for medical investigation, security (i.e. concealed weapon detection), and environmental monitoring. The basic concept is the same exploited by Charge Coupled Device (CCD) in a digital 


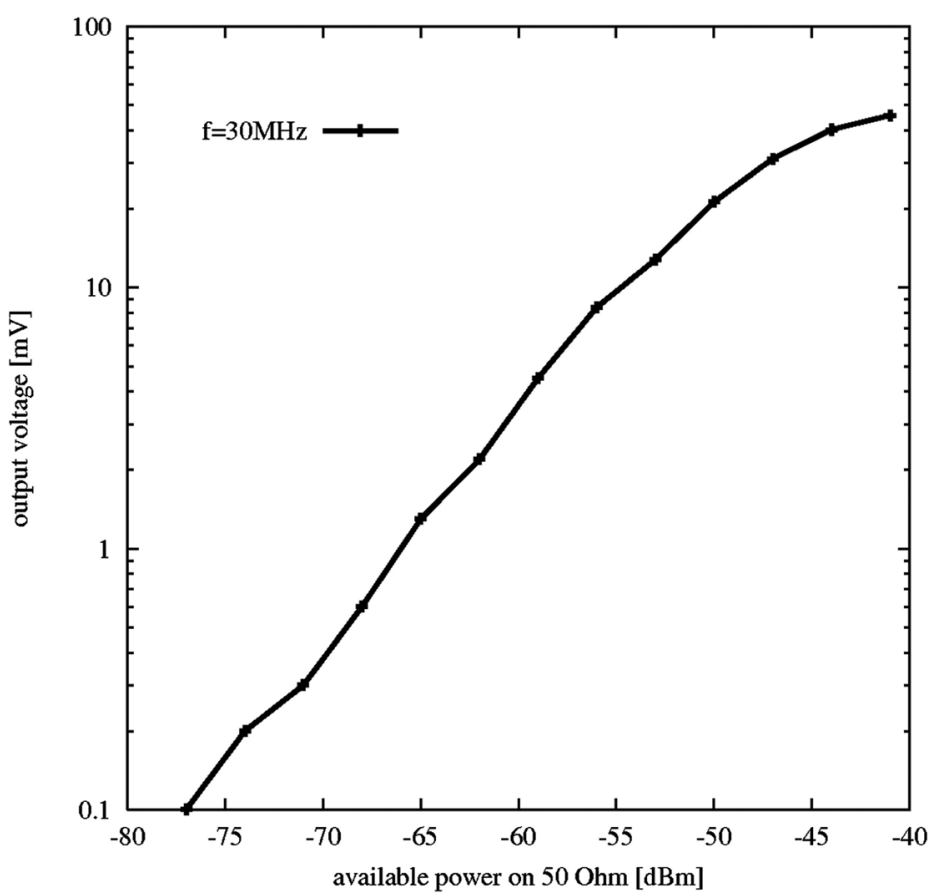

FIGURE 29 Output voltage of the square-low detector vs. input power (measured).

camera. The overall radiometric image is built up by the detections of each unit cell (i.e. the elementary integrated radiometric receiver) of the array. In the case of an array-based approach, it is interesting to consider the opportunity of exploiting direct detection instead of the down-conversion detection of the total power architecture. In fact, the direct detection architecture does not require any down-conversion mixer and, therefore, any distributed network for the local oscillation signal distribution [35]. However, the experiments on direct and down-conversion detections reported therein [35] show that such radiometer architecture introduces some design challenges in the amplifier and detector stages, both for the high-gain and high-selectivity requirements. As for a wearable sensor, it is worth citing the application to the civil safeguard, and in particular, for the improved safety of emergency operators [36]. In addition, it is worth considering other new interesting perspectives given by the opportunity of exploiting the detections by several system-on-chip radiometric sensors operating in different frequency bands (i.e. wavelength diversity). This multi-wavelength scan could allow us to build up more high-resolution radiometric images obtained by merging two or more images detected from the same scenario under observation where sources possess different characteristic emissions [37]. Such an approach is not only advisable, but also supported by the trend toward higher cutoff frequency of transistors.

\section{References}

[1] "New public safety applications and broadband internet access among uses envisioned by FCC authorization of ultra-wideband technology," Federal Communications Commission, 2002. [Online]. Available: http://www.fcc.gov/Bureaus/Engineering_Technology/News_Releases/2002/ nret0203.html

[2] “47 CFR Part 15,” Federal Communications Commission, 2002. [Online]. Available: http://www.fcc. gov/oet/info/rules/ 
[3] "Report summary from UWB radio systems committee," Ministry of Internal Affairs and Communications (MIC), 2006. [Online]. Available: http://www.soumu.go.jp/johotsusin/eng/pdf/060327 UWB report.pdf

[4] "Etsi ultra wide band," European Telecommunications Standards Institute (ETSI), 2008. [Online]. Available: http://www.etsi.org/WebSite/Technologies/UltraWideBand.aspx

[5] J.C. Lin, "Microwave sensing of physiological movement and volume change: a review," Bioelectromagnetics, vol. 13, pp. 557-565, 1992.

[6] A.D. Droitcurt, O. Boric-Lubecke, V.M. Lubecke, J. Lin, and G.T.A. Kovacs, "Range correlation and I/Q performance benefits in singlechip silicon radars for noncontact cardiopulmonary monitoring," IEEE Trans. Microwave Theory and Techniques, vol. 52, pp. 838-848, March 2004.

[7] T.E. McEwan, "Body monitoring and imaging apparatus and method," U.S. Patent 5,573,012, Nov. 12, 1996.

[8] I.J. Immoreev and S.V. Samkov, "Ultra-wideband (UWB) radar for remote measuring of main parameters of patient's vital activity," Radio Physics and Radio Astronomy, vol. 7, no. 4, pp. 404407, Dec. 2002.

[9] E.M. Staderini, "UWB radars in medicine," IEEE Aerospace and Electronic Systems Magazine, vol. 17, no. 1, pp. 13-18, Jan. 2002.

[10] S.F. Cleary, F. Nickless, L.M. Liu, and R. Hoffman, "Studies of exposure of rabbits to electromagnetic pulsed fields," Bioelectromagnetics, vol. 1, no. 3, pp. 345-352, 1980.

[11] J.A. D'Andrea, B.L. Cobb, and J.O. Lorge, "Lack of behavioural effects in the rhesus monkey: high peak microwave pulses at 1.3 GHZ," Bioelectromagnetics, vol. 10, no. 1, pp. 65-76, 1989.

[12] T.J. Walters, P. Mason, C. Sherry, C. Steffen, and J.H. Merritt, "No detectable bioeffects following acute exposure to high peak power ultrawide band electromagnetic radiation in rats," Aviation, Space, and Environmental Medicine, vol. 66, no. 6, pp. 562-567, June 1995.

[13] C.J. Sherry, D.W. Blick, T.J. Walters, and G.C. Brown, "Lack of behavioural effects in non-human primates after exposure to ultrawideband electromagnetic radiation in the microwave frequency range," Radiation Research, vol. 143, no. 1, pp. 93-97, July 1995.

[14] J. Merritt, J.L. Gel, and W.D. Hurt, "Considerations for human exposure standards for fast-rise-time high-peak-power electromagnetic pulses," Aviation, Space, and Environmental Medicine, vol. 6, no. 66, pp. 586-589, June 1995.

[15] J.R. Jauchem, R.L. Seaman, H.M. Lehnert, S.P. Mathur, K.L. Ryan, M.R. Frei, and W.D. Hurt, "Ultra-wideband electromagnetic pulses: lack of effects on heart rate and blood pressure during two-minute exposures of rats," Bioelectromagnetics, vol. 19, no. 5, pp. 330-333, 1998.

[16] M. Klemm and G. Troester, "Textile UWB antennas for wireless body area networks," IEEE Trans. Antennas and Propagation, vol. 54, no. 11, pp. 3192-3197, Nov. 2006.

[17] D. Zito, D. Pepe, B. Neri, D.D. Rossi, A. Lanata, A. Tognetti, and E.P. Scilingo, "Wearable systemon-a-chip UWB radar for health care and its application to the safety improvement of emergency operators," IEEE Intl. Conf. Engr. Medicine and Biology, Lyon, France, Aug. 2007, pp. 2651-2654.

[18] E. Della Mese, “Appunti al corso di Teoria e Tecnica Radar,” Ed. SEU Pisa.

[19] C. Gabriel and S. Gabriel, "Compilation of the dielectric properties of body tissues at RF and microwave frequencies," 2002, [Online].

Available: http://niremf.ifac.cnr.it/docs/DIELECTRIC/home.html

[20] D. Andreuccetti, R. Fossi, and C. Petrucci, "Calculation of the dielectric properties of body tissues in the frequency range $10 \mathrm{HZ}-100 \mathrm{GHZ}$, , 2002, [Online].

Available: http://niremf.ifac.cnr.it/tissprop/htmlclie/stfrtag

[21] H. Schantz, "Near field channel mode," IEEE P802.15 Working Group for Wireless Personal Area Networks, 2006.

[22] A. Monorchio, "Appunti di compatibilità elettromagnetica," draft 1.01. [Online]. Available: http://www2.ing.unipi.it/homepages/agostino.monorchio/emc.html

[23] K. Kiminami, A. Hirata, and T. Shiozawa, "Double-sided printed bowtie antenna for UWB communications," IEEE Antennas and Wireless Propagation Letters, vol. 3, pp. 152-153, 2004. 
[24] E. Staderini, "An UWB Radar Based Stealthy 'Lie Detector'," Online Technical Report, Available: www.hrvcongress.org/second/first/placed_3/Staderini_Art_Eng.pdf.

[25] K. Marsden, H.-J. Lee, D.S. Ha, and H.-S. Lee, "Low power CMOS reprogrammable pulse generator for UWB systems," IEEE Conf. Ultra Wideband Systems and Technologies, pp. 443-447, Nov. 2003.

[26] M.I. Skolnik, Radar Handbook, McGraw-Hill, 1970.

[27] D. Zito, D. Pepe, F. Zito, M. Mincica, D. De Rossi, "Wearable SoC UWB (3.1-10.6 GHz) radar for cardiopulmonary monitoring," IEEE Intl. Conf. VLSI-SoC, Rhodes, Greece, 13-15 October 2008.

[28] G. Luzi, P. Coppo, P. Ferrazzoli, S. Gagliani, T. Mazzoni, "Microwave radiometry as a tool for forest fire detection: model analysis and preliminary experiments," Microwave Radiometry and Remote Sensing of the Environment, D. Solimini ed., Utrecht, Netherlands, 1995, pp. 411-418.

[29] P.F. Goldsmith, Quasioptical Systems, IEEE Press, 1998.

[30] N. Skou, Microwave Radiometer Systems: Design and Analysis, Artech House, Norwood, MA, 1989.

[31] L. Fanucci, A. Hopper, B. Neri, D. Zito, "A novel fully integrated antenna switch for wireless systems," 33rd IEEE Conf. European Solid-State Device Research, 2003, pp. 553-556.

[32] F. Alimenti, S. Bonafoni, S. Leone, G. Tasselli, P. Basili, L. Roselli and K. Solbach, "Low-cost microwave radiometer for the detection of fire in forest environments", in IEEE Trans. Geoscience and Remote Sensing, vol. 46, no. 9, Sept. 2008.

[33] F.T. Ulaby, R.K. Moore and A.K. Fung, Microwave Remote Sensing, Artech House, 1981.

[34] F. Alimenti, D. Zito, A. Boni, M. Borgarino, A. Fonte, A. Carboni, et al., "System-on-chip microwave radiometer for thermal remote sensing and its application to the forest fire detection," IEEE Intl. Conf. Circuits and Systems, Malta, Aug. 31-Sept. 3, 2008.

[35] A. Fonte, D. Zito, F. Alimenti, "CMOS microwave radiometer: experiments on down-conversion and direct detections," IEEE Intl. Conf. Circuits and Systems, Malta, Aug. 31 - Sept. 3, 2008.

[36] D. Zito, F. Alimenti, A. Fonte, B. Neri, D. De Rossi, A. Lanata, A. Tognetti, "Wearable system-ona-chip radiometer for remote temperature sensing and its application to the safeguard of emergency operators," IEEE Intl. Conf. Engr. Medicine and Biology, Lyon, France, Aug. 2007, pp. 5751-5754.

[37] D.G. Gleed, R. Appleby, N.A. Salmon, S. Price, G.N. Sinclair, R.N. Anderton, J.R. Borrill, M.R.M. Wasley, and A.H. Lettington, "Operational issues for passive millimeter wave imaging systems," Proc. Intl. Society Optical Engineering (SPIE), vol. 3064, 1997, pp. 23-33. 
